\title{
Distribuição e conservação das aves na região do Cantão, Tocantins: ecótono Amazônia/Cerrado
}

\author{
Renato Torres Pinheiro ${ }^{1,3,4}$ \& Túlio Dornas ${ }^{2,3}$ \\ ${ }^{1}$ Universidade Federal do Tocantins - UFT, \\ Bloco BALA, sala 11c, Campus de Palmas, Palmas, TO, Brasil \\ ${ }^{2}$ Programa de Pós-graduação em Ciências do Ambiente, \\ Universidade Federal do Tocantins - UFT \\ ${ }^{3}$ Grupo de Pesquisa em Ecologia e Conservação de Aves do Tocantins, \\ Universidade Federal do Tocantins - UFT \\ ${ }^{4}$ Autor para correspondência: Renato Torres Pinheiro,e-mail: renatopin@uft.edu.br
}

PINHEIRO, R.T. \& DORNAS, T. 2009. Bird distribution and conservation on Cantão region, State of Tocatins: Amazon/Cerrado ecotone. Biota Neotrop. 9(1): http://www.biotaneotropica.org.br/v9n1/en/abstract?inventory+ bn02609012009.

\begin{abstract}
The Cantão region is a complex transition between Amazonia and Cerrado. Human interference is widespread even though the region is covered by two protected areas: the Cantão State Park and the Bananal Island/Cantão Environmental Protection Area. Bird inventories identified 418 species in the region, 382 on the present study. Of those, 27 are Amazonian and 9 Cerrado endemics. Four bird species are listed in the IUCN's red list as vulnerable: Penelope ochrogaster, Harpyhaliaetus coronatus, Cercomacra ferdinandi and Synallaxis simoni. This study also added eight species to the Cerrado biome list. The Cantão region is important for ten nearctic migrant species, including Pluvialis dominica, Actites macularia, Falco peregrinus and Progne subis. Habitat loss and deterioration caused by unsustainable tourism within the State Park, as well as cattle ranching and agriculture expansion within the Environmental Protected Area may have a strong impact on the populations of several bird species. We suggest that management actions should be focused on improving management and increasing coverage of protected areas to encompass marshlands and the Ipucas (natural forest patches) that are still well preserved in the region.
\end{abstract}

Keywords: bird conservation, Tocantins, Araguaia river valley.

PINHEIRO, R.T. \& DORNAS, T. 2009. Distribuição e conservação das aves na região do Cantão, Tocantins: ecótono Amazônia/Cerrado. Biota Neotrop. 9(1): http://www.biotaneotropica.org.br/v9n1/pt/abstract?invento ry+bn02609012009.

Resumo: A região do Cantão é uma transição entre a Amazônia e o Cerrado. Mesmo com a presença de duas unidades de conservação, o Parque Estadual do Cantão e a Área de Proteção Ambiental Ilha do Bananal/Cantão, a interferência humana é intensa. Inventários avifaunísticos realizados nestas áreas identificaram 418 espécies, sendo 382 neste estudo. Destas, 27 são endêmicas amazônicas e 9 do Cerrado. Quatro espécies são consideradas vulneráveis de extinção: Penelope ochrogaster, Harpyhaliaetus coronatus, Cercomacra ferdinandi e Synallaxis simoni. Este estudo também adiciona ainda oito novas espécies para a lista total de espécies do bioma Cerrado. A região também se destaca pela presença de migratórias neárticas, sendo identificadas dez espécies, entre elas, Pluvialis dominica, Actites macularia, Falco peregrinus e Progne subis. Ainda que as unidades de conservação locais exerçam um importante papel na conservação destas espécies, diversas ameaças colocam em risco suas populações, principalmente o turismo desorganizado no Parque e a expansão da agropecuária na APA. Sugere-se a realização de ações de manejo e a ampliação das áreas de conservação priorizando as florestas, varjões e Ipucas (capões de mata natural) ainda bem preservados na região.

Palavras-chave: conservação de aves, Tocantins, vale do rio Araguaia. 


\section{Introdução}

A região do Cantão, situada no centro-oeste do Estado do Tocantins, é um ecótono complexo, formado pela confluência de dois grandes biomas, Floresta Amazônica e Cerrado. A presença de inúmeros lagos e do regime de enchentes faz com que a região tenha ainda, características semelhantes à do Bioma Pantanal, formando um mosaico de ecossistemas muito peculiares e únicos na região. A singularidade e riqueza desses ecossistemas foram reconhecidos com a criação de duas Unidades de Conservação, o Parque Estadual do Cantão (PEC), que corresponde às áreas de vegetação inundada situada entre os rios Javaés/Araguaia e Côco e a Área de Proteção Ambiental Ilha do Bananal/Cantão (APA), caracterizada por formações vegetais típicas do Cerrado com elementos de Floresta Ombrófila representativos do Bioma Amazônico (Santos \& Lolis 2007).

Ainda que legalmente protegidas, as áreas naturais do Cantão vem sendo alteradas sistematicamente. A interferência humana é mais acentuada na APA, onde o relevo plano coberto em grande parte por vegetação de Cerrado, favorece o desenvolvimento agropecuário. Por outro lado, as áreas de vegetação mais densa com características amazônicas, têm sido selecionadas para programas de reforma agrária. Dentro do PEC, ainda que o regime de cheias limite o uso do solo, as culturas de subsistência tem promovido alterações na biota local e o turismo desordenado interferido na dinâmica de algumas espécies de aves ribeirinhas.

Iniciativas visando à conservação da biodiversidade local foram propostas pelo IBAMA, através do Corredor Ecológico AraguaiaBananal (http://www.ibama.gov.br/ecossistemas/projetosCerrado. htm) e da Conservação Internacional do Brasil em parceria com o Instituto Ecológica, que iniciou estudos preliminares para implantação do Corredor de Biodiversidade do Araguaia (http://www.conservation. org.br/onde/cerrado/index.php).

Do ponto de vista ornitológico a região da Ilha do Bananal foi escassamente explorada (Snethlage 1928, Hidasi 1968, 1983, 1998), porém, em função da criação das Unidades de Conservação locais, foram encontradas na APA Ilha do Bananal/Cantão 195 espécies de aves (MRS 1999), 217 no Parque Nacional do Araguaia (MMA/Eletronorte/Proaves 2001) e 317 espécies no P. E. Cantão (Tocantins 2004b), demonstrando a riqueza da avifauna local e o valor de conservação desta região.

O Cantão está inserido em uma região que apresenta uma elevada riqueza de aves e grande diversidade de ambientes, sendo considerada como prioritária para a conservação (MMA/SBF 2002). Sua destacada importância ecológica se deve ainda a outros fatores, tais como a presença de espécies endêmicas (Silva \& Bates 2002), ameaçadas de extinção (MMA 2003, IUCN 2006) e à destruição de áreas naturais do Cerrado (Klink \& Machado 2005).

Visando preencher algumas lacunas do conhecimento ornitológico do Estado do Tocantins e das áreas de transição entre o Cerrado e a Amazônia, o presente trabalho apresenta uma lista comentada das espécies que ocorrem dentro do Parque Estadual do Cantão e seu entorno imediato, na Área de Proteção Ambiental Ilha do Bananal/Cantão, enfatizando a riqueza avifaunística local e sua importância para a conservação da biodiversidade regional.

\section{Material e Métodos}

\section{1. Área de estudo}

O estudo foi realizado no Parque Estadual do Cantão e em três municípios da Área de Proteção Ambiental Ilha do Bananal/Cantão (Figura 1). A altitude média na região é de 150 m, o clima é úmido com

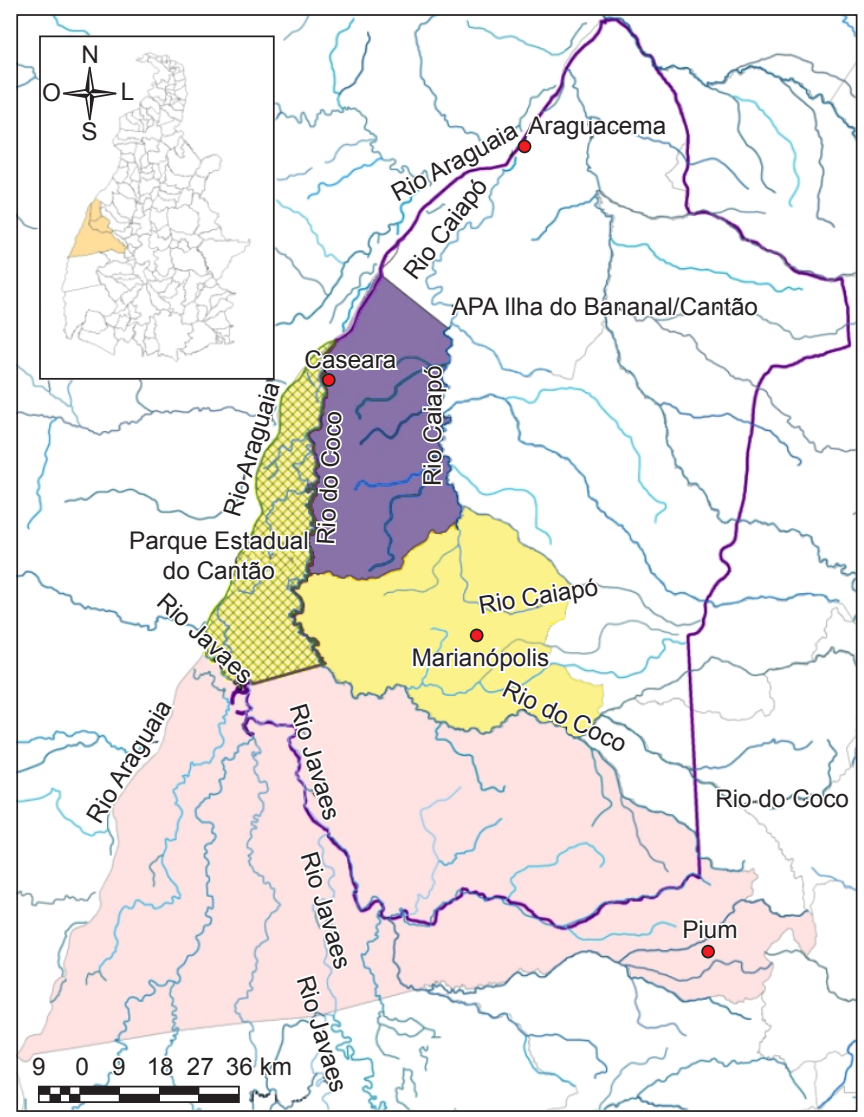

Figura 1. Localização do Parque Estadual do Cantão e dos limites da Área de Proteção Ambiental Ilha do Bananal/Cantão, com destaque para os três municípios onde se realizou o estudo.

Figure 1. Cantão State Park location and Bananal Island/Cantão Environmental Protection Area limits, detaching the three municipalities inventoried.

moderada ou nula deficiência hídrica, temperatura media de $28{ }^{\circ} \mathrm{C}$ e pluviosidade média anual de $2.100 \mathrm{~mm}$. (SEPLAN 2005).

Parque Estadual do Cantão (PEC): localizado no município de Pium $\left(9^{\circ}\right.$ a $10^{\circ} \mathrm{S}$ e $\left.50^{\circ} 10^{\prime} \mathrm{W}\right)$, possui uma extensão de 90.000 ha. $\mathrm{O}$ PEC é circundado por três rios: o Javaés ao sul, o Araguaia a oeste e o Côco a leste, sendo que os dois últimos confluem no extremo norte do PEC. A região onde o Parque está inserido é marcada por uma forte sazonalidade climática. No período chuvoso (outubro a abril) toda a planície é alagada, havendo um aumento de 7 a 10 m no nível da água dos rios. No período de estiagem (maio a setembro), o clima é seco, promovendo significativa redução do nível das águas, fazendo emergir vários bancos de areia nos leitos dos canais e rios da região (Tocantins 2004b). No Parque foram definidos cinco ecossistemas naturais: águas interiores (lagos e rios), ilhas fluviais, varjões, matas de terra firme (localmente denominada de mata de torrão) e matas de várzea. Detalhes da sua distribuição e vegetação estão disponíveis em Tocantins (2004a).

Área de Proteção Ambiental Ilha do Bananal/Cantão (APA): abrange aproximadamente 1.700.000 ha nos municípios de Abreulândia, Caseara, Pium, Marianópolis, Divinópolis, Araguacema, Dois Irmãos, Monte Santo e Chapada da Areia. Entre os objetivos de criação da APA está a ordenação do uso e ocupação do entorno do Parque Estadual do Cantão (MRS 1999). O extrativismo, agricultura e a pecuária extensiva são as principais atividades econômicas na região. A pecuária é a atividade mais expressiva, sendo desen- 
volvida principalmente em pastagens semi-naturais, entretanto, a baixa fertilidade do solo faz com que estas sejam abandonadas após alguns anos de pastoreio. Foram selecionados para este estudo, três dos nove municípios da APA, correspondendo àqueles localizados imediatamente no entorno do PEC e que abrangem toda sua extensão: municípios de Pium, Marianópolis e Caseara (Figura 1).

No município de Caseara foram amostradas as seguintes loca-

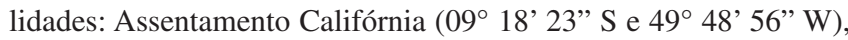
Assentamento Buritirana ( $09^{\circ} 27^{\prime} 46^{\prime \prime} \mathrm{S}$ e $\left.49^{\circ} 42^{\prime} 78^{\prime \prime} \mathrm{W}\right)$, Assenta-

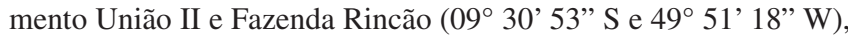
no município de Marianópolis o Assentamento Manchete (09 46' 75" S e 49 55'03" W) e no município de Pium a Fazenda Brasil Palmeira (10 04' $44^{\prime \prime}$ S e $\left.49^{\circ} 44^{\prime} 30^{\prime \prime} \mathrm{W}\right)$. Nestas localidades foram amostradas formações florestais compostas por Mata de Galeria, Floresta Aluvial, Floresta Ombrófila Aberta, Floresta Estacional Semidecidual, Cerradão e formações savânicas de Cerrado sentido restrito e Campo Cerrado, bem como áreas alteradas compostas por capoeiras, pastagens e monoculturas. Detalhes da vegetação foram descritos por Santos \& Lolis (2007). No texto a abreviação APA fará referência apenas aos três municípios amostrados no presente trabalho.

\section{Métodos}

O inventário da avifauna da região do Cantão foi realizado entre maio de 2004 e outubro de 2006. Na Área de Proteção Ambiental Ilha do Bananal/Cantão, a avifauna foi amostrada respectivamente entre 02 e 07 de maio, 08 e 13 de agosto de 2004 e 10 a 14 de maio de 2005. Foram utilizados dois métodos distintos e complementares: a) censos por observação direta realizados nas primeiras três horas da manhã e três horas no final da tarde, caminhando por estradas e trilhas, anotando-se todas as espécies visualizadas (binóculos Baush \& Lomb $10 \times 42$ ) ou ouvidas. Registros das vocalizações foram feitos com gravador digital Marantz PMD 670 equipado com microfone Sennheiser ME-66. Em algumas ocasiões utilizou-se a técnica do "playback" para atração e identificação das espécies menos conhecidas; b) redes de neblina foram utilizadas para captura e identificação de espécies pouco conspícuas. Em cada uma das cinco localidades, foram colocadas de 5 a 10 redes de 7-12 $\mathrm{m} \times 2,5 \mathrm{~m}$, as quais permaneceram abertas por um período de 6 horas, a partir do nascer do sol, totalizando $18 \mathrm{~h} /$ rede para cada ambiente.

No Parque Estadual do Cantão, a coleta de dados foi realizada com uma freqüência quinzenal, por doze meses consecutivos, entre outubro de 2005 e outubro de 2006 , em cada um dos cinco ambientes mencionados anteriormente (Tocantins 2004a), totalizando 24 censos em cada um deles. Os métodos e meios de amostragem variaram entre os ambientes terrestres e aquáticos: a) censos terrestres, realizados em transectos de dois mil metros de extensão seguindo o mesmo padrão descrito para a APA; b) redes de neblina foram colocadas em todos os ecossistemas (exceto rios e lagos), do mesmo modo que na APA, totalizando, neste caso, $20 \mathrm{~h} /$ rede para cada ambiente; c) censos aquáticos, realizados através de barco com motor de popa, nos principais cursos d'água do Parque (rios Araguaia, Javaés e Côco), percorrendo-se transectos de oito mil metros cada. Em ambos os métodos os censos foram realizados a partir do nascer do sol, anotando-se todas as espécies visualizadas (binóculos Pentax 8 × 40) ou ouvidas. A documentação das espécies ocorreu por meio de registro fotográfico digital (Konica Minolta Z13) e gravação das vocalizações (Marantz PMD 670).

A abundância relativa de cada espécie foi definida, segundo sua presença nos levantamentos realizados em cada habitat, seguindo o método descrito por Hilty \& Brown (1986) e Naka et al. (2002), onde, a frequiência de ocorrência para cada espécie foi calculada a partir do número de levantamentos em que uma determinada espécie foi detectada, dividida pelo número de levantamentos realizados em cada hábitat, como segue: Comum (C): espécies registradas entre $100 \%$ e $75 \%$ das visitas; Relativamente Comum (RC): espécies registradas entre $74 \%$ e $50 \%$ das visitas à campo; Incomuns (I): espécies registradas entre $49 \%$ e $25 \%$ das visitas à campo; Raras (R): espécies registradas em menos de $25 \%$ das visitas aos ambientes apropriados; Extremamente Raras (ER): espécies registradas em 1 ou 2 vezes nos ambientes e épocas apropriadas. Assumiu-se ainda que, os registros efetuados fora dos censos e capturas foram considerados como raros ou extremamente raros na região do Cantão.

Os hábitats preferenciais no qual cada espécie ocorre foi baseado em Olmos et al. (2004), sendo classificadas como: espécies estritamente florestais (F1), florestais que também utilizam áreas abertas (F2), estritamente campestres (C1), campestres que utilizam ambientes florestais (C2) e espécies aquáticas (A). A nomenclatura seguiu a Lista das Aves do Brasil, proposta pelo Comitê Brasileiro de Registros Ornitológicos (CBRO 2007).

\section{Resultados}

\section{Composição, distribuição e preferência de hábitat}

Foram identificadas 382 espécies referentes a 66 famílias, sendo 325 espécies no Parque Estadual do Cantão e 298 nos três municípios amostrados da Área de Proteção Ambiental Ilha do Bananal/Cantão. Deste total, 83 espécies foram encontradas apenas no PEC e 59 delas ocorreram exclusivamente na APA (Tabela 1).

A abundância relativa das espécies no Parque Estadual do Cantão indica que $16,0 \%$ das espécies são comuns, $27,7 \%$ relativamente comuns, $28,3 \%$ incomuns, $16,0 \%$ raras e $12,0 \%$ extremamente raras. Na APA, 8,9\% das espécies são comuns, $24,0 \%$ relativamente comuns, $36,0 \%$ incomuns, $29,1 \%$ raras e $2,0 \%$ extremamente raras (Tabela 1).

As espécies foram em sua maioria espécies florestais $(51,8 \%)$, das quais 103 foram consideradas estritamente florestais e 96 florestais que também usam áreas abertas. Um total de 122 espécies é campestre, das quais 41 são estritamente campestres e 81 campestres que também frequientam áreas florestais. Por fim, há 63 espécies aquáticas (Tabela 1).

A região do Cantão abriga um grande número de espécies endêmicas, das quais 27 foram consideradas endemismos amazônicos (ENA) (Stotz et al. 1996) e nove endemismos do Cerrado (ENC) (Silva 1997, Silva \& Santos 2005). Isto representa 9,8\% dos endemismos amazônicos e $30 \%$ dos endemismos de Cerrado. No que se refere às endêmicas do Cerrado, o PEC apresentou cinco espécies, enquanto na APA foram seis. Em relação às espécies endêmicas da Amazônia, no PEC encontrou-se 26 espécies contra 13 da APA (Tabela 1).

Dentre as espécies migratórias, dez foram consideradas visitantes do Hemisfério Norte, Pandion haliaetus, Buteo swainsoni, Falco peregrinus, Pluvialis dominica, Tringa flavipes, Tringa solitaria, Actitis macularius, Calidris fuscicollis, Progne subis e Hirundo rustica (Rapolle et al. 1993, Stotz et al. 1996, Sick 1997, 1983, CBRO 2007).

\section{Status de Conservação}

No Cantão encontramos nove espécies com algum grau de ameaça, sendo quatro vulneráveis (VU): Penelope ochrogaster, Harpyhaliaetus coronatus, Cercomacra ferdinandi e Synallaxis simoni (MMA, 2003) e cinco quase ameaçadas (NT): Rhea americana, Neochen jubata, Harpya harpyja, Alipiopsitta xanthopis e Charitospiza eucosma, segundo o MMA (2003) e IUCN (2006).

Rhea americana (Linnaeus, 1758) - Para a área do Parque Estadual do Cantão esta espécie é conhecida através de dois registros, 
Tabela 1. Espécies de aves registradas no Parque Estadual do Cantão e Área de Proteção Ambiental Ilha do Bananal/Cantão, centro-oeste do Estado do Tocantins. Table 1. Bird species recorded in the Cantão State Park and Bananal Island/Cantão Environmental Protection Area, west-central Tocantins State.

\section{Táxons/abundância relativa \\ APA Ilha do Bananal/ Parque Estadual Habitat \\ Status Cantão do Cantão}

Rheidae Bonaparte, 1849

Rhea americana (Linnaeus, 1758) ${ }^{1}$

I

Tinamidae Gray, 1840

Tinamus tao Temminck, 1815*

Crypturellus cinereus (Gmelin, 1789)*

Crypturellus soui (Hermann, 1783) ${ }^{1 \mathrm{~A}}$

Crypturellus undulatus (Temminck, 1815) ${ }^{1}$

Crypturellus parvirostris (Wagler, 1827) ${ }^{1}$

Rhynchotus rufescens (Temminck, 1815) ${ }^{1 \mathrm{~A}}$

Anhimidae Stejneger, 1885

Anhima cornuta (Linnaeus, 1766) ${ }^{1}$

Anatidae Leach, 1820

Dendrocygna viduata (Linnaeus, 1766) ${ }^{1}$

Dendrocygna autumnalis (Linnaeus, 1758) ${ }^{1}$

Neochen jubata (Spix, 1825) ${ }^{1}$

Cairina moschata (Linnaeus, 1758) ${ }^{1}$

Amazonetta brasiliensis (Gmelin, 1789) ${ }^{1}$

Cracidae Rafinesque, 1815

Ortalis (cf) motmot (Linnaeus, 1766) ${ }^{1}$

Penelope superciliaris Temminck, $1815^{1}$

Penelope pileata Wagler, $1830^{2}$

Penelope ochrogaster Pelzeln, $1870^{1}$

Crax fasciolata Spix, $1825^{1}$

Phalacrocoracidae Reichenbach, 1849

Phalacrocorax brasilianus (Gmelin, 1789) ${ }^{1}$

Anhingidae Reichenbach, 1849

Anhinga anhinga (Linnaeus, 1766) ${ }^{1}$

Ardeidae Leach, 1820

Tigrisoma lineatum (Boddaert, 1783) ${ }^{1}$

Agamia agami (Gmelin, 1789) ${ }^{1 *}$

Cochlearius cochlearius (Linnaeus, 1766) ${ }^{1}$

Nycticorax nycticorax (Linnaeus, 1758) ${ }^{1}$

Butorides striata (Linnaeus, 1758) ${ }^{1}$

Bubulcus ibis (Linnaeus, 1758) ${ }^{1}$

Ardea cocoi Linnaeus, $1766^{1}$

Ardea alba Linnaeus, $1758^{1}$

Syrigma sibilatrix (Temminck, 1824)

Pilherodius pileatus (Boddaert, 1783)1

Egretta thula (Molina, 1782) ${ }^{1}$

Egretta caerulea (Linnaeus, 1758)

Threskiornithidae Poche, 1904

Mesembrinibis cayennensis (Gmelin, 1789) ${ }^{1}$

Phimosus infuscatus (Lichtenstein, 1823) ${ }^{1}$

Theristicus caudatus (Boddaert, 1783) ${ }^{1}$

Platalea ajaja Linnaeus, $1758^{1}$

Ciconiidae Sundevall, 1836

Jabiru mycteria (Lichtenstein, 1819) ${ }^{1}$

Mycteria americana Linnaeus, $1758^{1}$

Cathartidae Lafresnaye, 1839

\begin{tabular}{|c|c|c|c|}
\hline I & ER & $\mathrm{C} 1$ & $\mathrm{NT}^{\mathrm{B}}$ \\
\hline I & ER & F1 & - \\
\hline I & $\mathrm{RC}$ & $\mathrm{F} 1$ & ENA \\
\hline $\mathrm{RC}$ & $\mathrm{RC}$ & F1 & - \\
\hline $\mathrm{RC}$ & $\mathrm{C}$ & F1 & - \\
\hline $\mathrm{RC}$ & $\mathrm{RC}$ & $\mathrm{C} 2$ & - \\
\hline I & I & $\mathrm{C} 1$ & - \\
\hline $\mathrm{R}$ & I & $\mathrm{A}$ & - \\
\hline I & I & $\mathrm{A}$ & - \\
\hline I & I & A & - \\
\hline I & $\mathrm{RC}$ & A & $\mathrm{NT}^{\mathrm{A}, \mathrm{B}}$ \\
\hline I & $\mathrm{RC}$ & A & - \\
\hline $\mathrm{R}$ & $\mathrm{R}$ & A & - \\
\hline- & ER & - & - \\
\hline I & I & F2 & - \\
\hline- & - & & - \\
\hline $\mathrm{R}$ & $\mathrm{C}$ & $\mathrm{F} 1$ & $\mathrm{VU}^{\mathrm{A}, \mathrm{B}} \mathrm{ENC}$ \\
\hline I & $\mathrm{C}$ & $\mathrm{F} 1$ & - \\
\hline $\mathrm{RC}$ & $\mathrm{RC}$ & A & - \\
\hline $\mathrm{RC}$ & $\mathrm{C}$ & A & - \\
\hline I & $\mathrm{C}$ & A & - \\
\hline- & $\mathrm{R}$ & A & - \\
\hline $\mathrm{R}$ & $\mathrm{R}$ & A & - \\
\hline $\mathrm{R}$ & I & A & - \\
\hline $\mathrm{RC}$ & $\mathrm{C}$ & A & - \\
\hline $\mathrm{RC}$ & I & C2 & - \\
\hline $\mathrm{RC}$ & $\mathrm{C}$ & A & - \\
\hline I & $\mathrm{RC}$ & A & - \\
\hline $\mathrm{R}$ & - & $\mathrm{A}$ & - \\
\hline I & $\mathrm{R}$ & A & - \\
\hline I & $\mathrm{RC}$ & A & - \\
\hline- & ER & A & - \\
\hline $\mathrm{RC}$ & $\mathrm{RC}$ & F2 & - \\
\hline $\mathrm{R}$ & $\mathrm{RC}$ & A & - \\
\hline $\mathrm{RC}$ & $\mathrm{RC}$ & $\mathrm{C} 2$ & - \\
\hline I & $\mathrm{RC}$ & A & - \\
\hline I & I & $\mathrm{A}$ & - \\
\hline I & $\mathrm{RC}$ & $\mathrm{A}$ & - \\
\hline
\end{tabular}


Tabela 1. Continuação...

\begin{tabular}{|c|c|c|c|c|}
\hline Táxons/abundância relativa & $\begin{array}{c}\text { APA Ilha do Bananal/ } \\
\text { Cantão }\end{array}$ & $\begin{array}{c}\text { Parque Estadual } \\
\text { do Cantão }\end{array}$ & Habitat & Status \\
\hline Cathartes aura (Linnaeus, 1758) ${ }^{1}$ & $\mathrm{C}$ & $\mathrm{RC}$ & $\mathrm{C} 2$ & - \\
\hline Cathartes burrovianus Cassin, $1845^{1}$ & I & $\mathrm{R}$ & $\mathrm{C} 2$ & - \\
\hline Coragyps atratus (Bechstein, 1793) ${ }^{1}$ & $\mathrm{RC}$ & $\mathrm{RC}$ & $\mathrm{C} 2$ & - \\
\hline Sarcoramphus papa (Linnaeus, 1758) & $\mathrm{R}$ & ER & $\mathrm{F} 2$ & - \\
\hline \multicolumn{5}{|l|}{ Pandionidae Bonaparte, 1854} \\
\hline Pandion haliaetus (Linnaeus, 1758) ${ }^{1}$ & ER & I & A & VN \\
\hline \multicolumn{5}{|l|}{ Accipitridae Vigors, 1824} \\
\hline Elanoides forficatus (Linnaeus, 1758) ${ }^{1}$ & $\mathrm{R}$ & $\mathrm{R}$ & $\mathrm{F} 2$ & - \\
\hline Gampsonyx swainsonii Vigors, $1825^{1}$ & $\mathrm{R}$ & $\mathrm{R}$ & $\mathrm{F} 2$ & - \\
\hline Elanus leucurus (Vieillot, 1818) ${ }^{1}$ & $\mathrm{R}$ & - & $\mathrm{C} 1$ & - \\
\hline Rostrhamus sociabilis (Vieillot, 1817) ${ }^{1}$ & I & $\mathrm{R}$ & A & - \\
\hline Ictinia plumbea $\left(\right.$ Gmelin, 1788) ${ }^{1}$ & $\mathrm{R}$ & - & $\mathrm{F} 2$ & - \\
\hline Circus buffoni (Gmelin, 1788) & - & ER & $\mathrm{F} 2$ & - \\
\hline Accipiter superciliosus (Linnaeus, 1766) & ER & ER & $\mathrm{F} 2$ & - \\
\hline Geranospiza caerulescens (Vieillot, 1817) ${ }^{1}$ & - & I & $\mathrm{F} 2$ & - \\
\hline Buteogallus urubitinga $\left(\right.$ Gmelin, 1788) ${ }^{1}$ & I & $\mathrm{RC}$ & $\mathrm{F} 2$ & - \\
\hline Heterospizias meridionalis (Latham, 1790) ) $^{1 \mathrm{~A}}$ & I & ER & $\mathrm{C} 2$ & - \\
\hline Micrastur ruficollis (Vieillot, 1817) ${ }^{2}$ & - & - & - & - \\
\hline Micrastur semitorquatus (Vieillot, 1817) ${ }^{2}$ & - & - & - & - \\
\hline Harpyhaliaetus coronatus (Vieillot, 1817) & ER & ER & $\mathrm{F} 2$ & $\mathrm{VU}^{\mathrm{A}, \mathrm{B}}$ \\
\hline Busarellus nigricollis (Latham, 1790) ${ }^{1}$ & I & $\mathrm{RC}$ & A & - \\
\hline Rupornis magnirostris (Gmelin, 1788) ${ }^{1}$ & $\mathrm{C}$ & $\mathrm{C}$ & $\mathrm{C} 2$ & - \\
\hline Buteo albicaudatus Vieillot, 1816 & $\mathrm{R}$ & - & $\mathrm{C} 2$ & - \\
\hline Buteo nitidus (Latham, 1790) & - & ER & $\mathrm{F} 2$ & - \\
\hline Buteo swainsoni Bonaparte, 1838 & - & $\mathrm{RC}$ & $\mathrm{C} 2$ & $\mathrm{VN}$ \\
\hline Buteo albonotatus Kaup, $1847^{1}$ & $\mathrm{R}$ & ER & $\mathrm{C} 1$ & - \\
\hline Harpia harpyja (Linnaeus, 1758) & - & ER & $\mathrm{F} 1$ & $\mathrm{NT}^{\mathrm{B}}$ \\
\hline Spizaetus tyrannus (Wied, 1820) ${ }^{1 *}$ & - & ER & $\mathrm{F} 1$ & - \\
\hline Spizaetus ornatus (Daudin, 1800) ${ }^{1}$ & - & ER & $\mathrm{F} 1$ & - \\
\hline \multicolumn{5}{|l|}{ Falconidae Leach, 1820} \\
\hline Daptrius ater Vieillot, $1816^{1 *}$ & - & $\mathrm{R}$ & $\mathrm{F} 2$ & - \\
\hline Ibycter americanus (Boddaert, 1783) ${ }^{1}$ & $\mathrm{R}$ & ER & $\mathrm{F} 2$ & - \\
\hline Caracara plancus (Miller, 1777) ${ }^{1}$ & $\mathrm{C}$ & $\mathrm{C}$ & $\mathrm{C} 2$ & - \\
\hline Milvago chimachima (Vieillot, 1816) & $\mathrm{C}$ & I & $\mathrm{C} 2$ & - \\
\hline Herpetotheres cachinnans (Linnaeus, 1758) ${ }^{1}$ & I & $\mathrm{RC}$ & $\mathrm{F} 2$ & - \\
\hline Falco sparverius Linnaeus, $1758^{1}$ & $\mathrm{RC}$ & I & $\mathrm{C} 1$ & - \\
\hline Falco rufigularis Daudin, $1800^{1}$ & $\mathrm{R}$ & $\mathrm{R}$ & $\mathrm{F} 2$ & - \\
\hline Falco femoralis Temminck, 1822 & I & - & $\mathrm{C} 1$ & - \\
\hline Falco peregrinus Tunstall, 1771 & - & ER & $\mathrm{C} 1$ & VN \\
\hline \multicolumn{5}{|l|}{ Aramidae Bonaparte, 1852} \\
\hline Aramus guarauna (Linnaeus, 1766) ${ }^{1}$ & $\mathrm{R}$ & $\mathrm{RC}$ & A & - \\
\hline \multicolumn{5}{|l|}{ Rallidae Rafinesque, 1815} \\
\hline Aramides ypecaha (Vieillot, 1819) & $\mathrm{R}$ & $\mathrm{R}$ & A & - \\
\hline Aramides cajanea (Statius Muller, 1776) ${ }^{1}$ & $\mathrm{RC}$ & $\mathrm{RC}$ & A & - \\
\hline Laterallus viridis (Statius Muller, 1776) ${ }^{1}$ & - & $\mathrm{R}$ & A & - \\
\hline Laterallus exilis (Temminck, 1831) ${ }^{2}$ & - & - & - & - \\
\hline Porzana albicollis (Vieillot, 1819) ${ }^{1}$ & - & I & A & - \\
\hline Gallinula chloropus (Linnaeus, 1758) & $\mathrm{R}$ & - & A & - \\
\hline Porphyrio martinica (Linnaeus, 1766) ${ }^{1}$ & - & $\mathrm{R}$ & A & - \\
\hline Porphyrio flavirostris (Gmelin, 1789) ${ }^{1}$ & - & $\mathrm{I}$ & $\mathrm{A}$ & - \\
\hline
\end{tabular}


Tabela 1. Continuação...

\section{Táxons/abundância relativa}

Heliornis fulica (Boddaert, 1783)

Eurypygidae Selby, 1840

Eurypyga helias (Pallas, 1781) ${ }^{1 *}$

Cariamidae Bonaparte, 1850

Cariama cristata (Linnaeus, 1766)

Jacanidae Chenu \& Des Murs, 1854

Jacana jacana (Linnaeus, 1766) ${ }^{1}$

Charadriidae Leach, 1820

Vanellus cayanus (Latham, 1790) ${ }^{1}$

Vanellus chilensis (Molina, 1782) ${ }^{1}$

Pluvialis squatarola (Linnaeus, 1758) ${ }^{2}$

Pluvialis dominica (Statius Muller, 1776)

Charadrius semipalmatus Bonaparte, $1825^{2}$

Charadrius collaris Vieillot, $1818^{1}$

Scolopacidae Rafinesque, 1815

Gallinago paraguaiae (Vieillot, 1816) ${ }^{1}$

Tringa flavipes (Gmelin, 1789) ${ }^{1}$

Tringa solitaria Wilson, $1813^{1}$

Actitis macularius (Linnaeus, 1766) ${ }^{1}$

Calidris fuscicollis (Vieillot, 1819)

Sternidae Vigors, 1825

Sternula superciliaris (Vieillot, 1819) ${ }^{1}$

Phaetusa simplex (Gmelin, 1789) ${ }^{1}$

Rynchopidae Bonaparte, 1838

Rynchops niger Linnaeus, $1758^{1}$

Columbidae Leach, 1820

Columbina passerina (Linnaeus, 1758)

Columbina minuta (Linnaeus, 1766) ${ }^{2}$

Columbina talpacoti (Temminck, 1811) ${ }^{1}$

Columbina squammata (Lesson, 1831) ${ }^{1}$

Claravis pretiosa (Ferrari-Perez, 1886) ${ }^{1}$

Uropelia campestris (Spix, 1825) ${ }^{1}$

Patagioenas speciosa (Gmelin, 1789) ${ }^{1}$

Patagioenas picazuro (Temminck, 1813) ${ }^{1}$

Patagioenas cayennensis (Bonnaterre, 1792) ${ }^{1}$

Patagioenas subvinacea (Lawrence, 1868) ${ }^{1 *}$

Zenaida auriculata (Des Murs, 1847)

Leptotila verreauxi Bonaparte, $1855^{1}$

Leptotila rufaxilla (Richard \& Bernard, 1792) ${ }^{1}$

Geotrygon montana (Linnaeus, 1758) ${ }^{1}$

Psittacidae Rafinesque, 1815

Ara ararauna (Linnaeus, 1758) ${ }^{1}$

Orthopsittaca manilata (Boddaert, 1783)

Primolius auricollis (Cassin, 1853) ${ }^{1}$

Primolius maracana (Vieillot, 1816) ${ }^{2 \mathrm{~A}}$

Diopsittaca nobilis (Linnaeus, 1758) ${ }^{1}$

Aratinga leucophthalma(Statius Muller, 1776) ${ }^{1}$

Aratinga aurea (Gmelin, 1788) ${ }^{1}$

Brotogeris chiriri (Vieillot, 1818) ${ }^{1}$

APA Ilha do Bananal/ Parque Estadual Habitat do Cantão

Cantão

$\mathrm{R}$

I

$\mathrm{RC}$

$\mathrm{RC}$

$\mathrm{RC}$

$\mathrm{RC}$

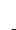

I

$\mathrm{R}$

$\mathrm{R}$
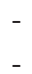

I

I

$\mathrm{R}$

-

C

C

I

I

I

$\mathrm{RC}$

$\mathrm{RC}$

$\mathrm{R}$

$\mathrm{R}$

$\mathrm{R}$

I

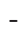

$\mathrm{R}$

-

$-$

I

I

C

I

A

$$
\text { I }
$$

F2

$\mathrm{RC}$

C1

A

A

A

-

R

-

$\mathrm{RC}$

A

A

A

VN

A $\quad \mathrm{VN}$

A $\quad \mathrm{VN}$

A

VN

$\mathrm{RC}$

C

A

A

C

A

C1

C2

$\mathrm{C} 2$

F2

C1

F2

C2

F2

F1

C1

F2

F2

F1

$\mathrm{R}$

$\mathrm{RC}$

$\mathrm{R}$

$\mathrm{RC}$

C2

F2

F2

$\mathrm{RC}$

C2

C2

C2

F2 
Tabela 1. Continuação...

\begin{tabular}{|c|c|c|c|c|}
\hline Táxons/abundância relativa & $\begin{array}{l}\text { APA Ilha do Bananal/ } \\
\text { Cantão }\end{array}$ & $\begin{array}{c}\text { Parque Estadual } \\
\text { do Cantão }\end{array}$ & Habitat & Status \\
\hline Alipiopsitta xanthops (Spix, 1824) & $\mathrm{R}$ & - & $\mathrm{C} 2$ & $\mathrm{NT}^{\mathrm{B}}, \mathrm{ENC}$ \\
\hline Pionus menstruus (Linnaeus, 1766) ${ }^{1 *}$ & I & I & F1 & - \\
\hline Amazona aestiva (Linnaeus, 1758$)^{1}$ & $\mathrm{R}$ & $\mathrm{R}$ & $\mathrm{C} 2$ & - \\
\hline Amazona amazonica (Linnaeus, 1766) ${ }^{1}$ & $\mathrm{C}$ & $\mathrm{C}$ & $\mathrm{F} 2$ & - \\
\hline \multicolumn{5}{|l|}{ Opisthocomidae Swainson, 1837} \\
\hline Opisthocomus hoazin (Statius Muller, 1776) ${ }^{1 *}$ & $\mathrm{RC}$ & $\mathrm{C}$ & $\mathrm{F} 1$ & - \\
\hline \multicolumn{5}{|l|}{ Cuculidae Leach, 1820} \\
\hline Piaya cayana (Linnaeus, 1766) ${ }^{1}$ & $\mathrm{RC}$ & I & $\mathrm{F} 2$ & - \\
\hline Cоссусиа minuta (Vieillot, 1817$)^{1 *}$ & $\mathrm{E}$ & $\mathrm{R}$ & $\mathrm{F} 2$ & - \\
\hline Crotophaga major Gmelin, $1788^{1}$ & I & $\mathrm{RC}$ & $\mathrm{F} 2$ & - \\
\hline Crotophaga ani Linnaeus, $1758^{1}$ & $\mathrm{C}$ & I & $\mathrm{C} 2$ & - \\
\hline Guira guira $(\text { Gmelin, } 1788)^{1}$ & $\mathrm{C}$ & I & $\mathrm{C} 2$ & - \\
\hline Tapera naevia (Linnaeus, 1766) ${ }^{1}$ & I & I & $\mathrm{F} 2$ & - \\
\hline Dromococcyx phasianellus (Spix, 1824) ${ }^{2}$ & - & - & - & - \\
\hline Dromococcyx pavoninus Pelzeln, $1870^{1}$ & - & ER & $\mathrm{F} 1$ & - \\
\hline \multicolumn{5}{|l|}{ Tytonidae Mathews, 1912} \\
\hline Tyto alba (Scopoli, 1769) ${ }^{1}$ & $\mathrm{R}$ & - & $\mathrm{C} 2$ & - \\
\hline \multicolumn{5}{|l|}{ Strigidae Leach, 1820} \\
\hline Megascops choliba (Vieillot, 1817) ${ }^{1}$ & $\mathrm{RC}$ & $\mathrm{RC}$ & $\mathrm{F} 2$ & - \\
\hline Megascops watsonii (Cassin, 1849)2 & - & - & - & - \\
\hline Lophostrix cristata (Daudin, 1800) ${ }^{2}$ & - & - & - & - \\
\hline Pulsatrix perspicillata $\left(\right.$ Latham, 1790) ${ }^{1}$ & - & I & $\mathrm{F} 1$ & - \\
\hline Bubo virginianus $\left(\right.$ Gmelin, 1788) ${ }^{1}$ & - & ER & $\mathrm{F} 1$ & - \\
\hline Strix huhula Daudin, $1800^{2}$ & - & - & - & - \\
\hline Glaucidium brasilianum (Gmelin, 1788) ${ }^{1}$ & I & I & $\mathrm{F} 2$ & - \\
\hline Athene cunicularia (Molina, 1782) & $\mathrm{RC}$ & I & $\mathrm{C} 2$ & - \\
\hline \multicolumn{5}{|l|}{ Nyctibiidae Chenu \& Des Murs, 1851} \\
\hline Nyctibius griseus (Gmelin, 1789) ${ }^{1}$ & $\mathrm{R}$ & $\mathrm{R}$ & $\mathrm{F} 2$ & - \\
\hline \multicolumn{5}{|l|}{ Caprimulgidae Vigors, 1825} \\
\hline Lurocalis semitorquatus (Gmelin, 1789)² & - & - & - & - \\
\hline Chordeiles rupestris (Spix, 1825) ${ }^{1}$ & - & $\mathrm{R}$ & $\mathrm{C} 1$ & - \\
\hline Chordeiles acutipennis (Hermann, 1783) & - & $\mathrm{R}$ & $\mathrm{C} 1$ & - \\
\hline Nyctiprogne leucopyga $\left(\right.$ Spix, 1825) ${ }^{1}$ & - & $\mathrm{RC}$ & $\mathrm{C} 2$ & - \\
\hline Nyctidromus albicollis (Gmelin, 1789) ${ }^{1}$ & $\mathrm{RC}$ & $\mathrm{C}$ & $\mathrm{F} 2$ & - \\
\hline Caprimulgus rufus Boddaert, 1783 & $\mathrm{R}$ & - & $\mathrm{F} 1$ & - \\
\hline Caprimulgus maculicaudus (Lawrence, 1862) ${ }^{2}$ & - & - & - & - \\
\hline Caprimulgus parvulus Gould, $1837^{1}$ & I & - & $\mathrm{C} 2$ & - \\
\hline Hydropsalis climacocerca (Tschudi, 1844) ${ }^{1}$ & I & $\mathrm{RC}$ & $\mathrm{C} 2$ & ENA \\
\hline Hydropsalis torquata $\left(\right.$ Gmelin, 1789) ${ }^{2}$ & - & - & - & - \\
\hline \multicolumn{5}{|l|}{ Apodidae Olphe-Galliard, 1887} \\
\hline Cypseloides senex (Temminck, 1826) & - & ER & $\mathrm{C} 2$ & - \\
\hline Chaetura spinicaudus $\left(\right.$ Temminck, 1839) ${ }^{1}$ & - & $\mathrm{R}$ & $\mathrm{C} 2$ & - \\
\hline Chaetura meridionalis Hellmayr, $1907^{1}$ & I & $\mathrm{R}$ & $\mathrm{C} 2$ & - \\
\hline Tachornis squamata $(\text { Cassin, } 1853)^{1 \mathrm{~A}}$ & I & $\mathrm{R}$ & $\mathrm{C} 2$ & - \\
\hline Panyptila cayennensis $\left(\right.$ Gmelin, 1789) ${ }^{1}$ & - & $\mathrm{R}$ & $\mathrm{C} 2$ & - \\
\hline \multicolumn{5}{|l|}{ Trochilidae Vigors, 1825} \\
\hline Glaucis hirsutus (Gmelin, 1788) ${ }^{1}$ & - & $\mathrm{R}$ & F1 & - \\
\hline Phaethornis maranhaoensis Grantsau, $1968^{2}$ & - & - & - & - \\
\hline Phaethornis nattereri Berlepsch, 1887 & - & $\mathrm{RC}$ & $\mathrm{F} 1$ & - \\
\hline Phaethornis ruber $(\text { Linnaeus, } 1758)^{1 \mathrm{~A}}$ & $\mathrm{RC}$ & $\mathrm{RC}$ & $\mathrm{F} 1$ & - \\
\hline
\end{tabular}


Tabela 1. Continuação...

\begin{tabular}{|c|c|c|c|c|}
\hline Táxons/abundância relativa & $\begin{array}{l}\text { APA Ilha do Bananal/ } \\
\text { Cantão }\end{array}$ & $\begin{array}{l}\text { Parque Estadual } \\
\text { do Cantão }\end{array}$ & Habitat & Status \\
\hline Phaethornis pretrei (Lesson \& Delattre, 1839) & $\mathrm{R}$ & $\mathrm{R}$ & $\mathrm{F} 2$ & - \\
\hline Eupetomena macroura $(\text { Gmelin, } 1788)^{1 \mathrm{~A}}$ & I & $\mathrm{R}$ & $\mathrm{F} 2$ & - \\
\hline Anthracothorax nigricollis (Vieillot, 1817) ${ }^{1}$ & $\mathrm{R}$ & $\mathrm{I}$ & $\mathrm{F} 2$ & - \\
\hline Chrysolampis mosquitus (Linnaeus, 1758) & $\mathrm{R}$ & - & $\mathrm{C} 2$ & - \\
\hline Lophornis gouldii (Lesson, 1832) & $\mathrm{R}$ & - & $\mathrm{C} 1$ & - \\
\hline Chlorostilbon mellisugus (Linnaeus, 1758)* & - & $\mathrm{R}$ & $\mathrm{C} 1$ & - \\
\hline Thalurania furcata (Gmelin, 1788) ${ }^{1}$ & I & I & $\mathrm{F} 2$ & - \\
\hline Hylocharis cyanus (Vieillot, 1818) ${ }^{2}$ & - & - & - & - \\
\hline Polytmus guainumbi (Pallas, 1764) ${ }^{1}$ & - & I & $\mathrm{C} 1$ & - \\
\hline Amazilia versicolor (Vieillot, 1818) & - & $\mathrm{I}$ & $\mathrm{F} 2$ & - \\
\hline Amazilia fimbriata $\left(\right.$ Gmelin, 1788) ${ }^{1}$ & $\mathrm{R}$ & $\mathrm{R}$ & $\mathrm{C} 2$ & - \\
\hline Heliomaster longirostris (Audebert \& Vieillot, 1801) ${ }^{1}$ & I & $\mathrm{R}$ & $\mathrm{F} 2$ & - \\
\hline \multicolumn{5}{|l|}{ Trogonidae Lesson, 1828} \\
\hline Trogon viridis Linnaeus, $1766^{1 *}$ & I & $\mathrm{RC}$ & F1 & - \\
\hline Trogon curucui Linnaeus, 1766 & I & $\mathrm{R}$ & $\mathrm{F} 1$ & - \\
\hline Trogon $(\mathrm{cf})$ violaceus Gmelin, 1788* & - & ER & $\mathrm{F} 1$ & - \\
\hline Trogon melanurus Swainson, $1838^{1 *}$ & I & $\mathrm{RC}$ & $\mathrm{F} 1$ & - \\
\hline \multicolumn{5}{|l|}{ Alcedinidae Rafinesque, 1815} \\
\hline Megaceryle torquata (Linnaeus, 1766) ${ }^{1}$ & $\mathrm{C}$ & $\mathrm{C}$ & A & - \\
\hline Chloroceryle amazona (Latham, 1790) ${ }^{1}$ & $\mathrm{RC}$ & $\mathrm{C}$ & A & - \\
\hline Chloroceryle americana (Gmelin, 1788) ${ }^{1}$ & $\mathrm{RC}$ & $\mathrm{RC}$ & A & - \\
\hline Chloroceryle inda (Linnaeus, 1766) ${ }^{1}$ & I & $\mathrm{RC}$ & A & - \\
\hline Chloroceryle aenea (Pallas, 1764) ${ }^{1}$ & I & $\mathrm{RC}$ & A & - \\
\hline \multicolumn{5}{|l|}{ Momotidae Gray, 1840} \\
\hline Momotus momota (Linnaeus, 1766) ${ }^{1}$ & I & I & $\mathrm{F} 1$ & - \\
\hline \multicolumn{5}{|l|}{ Galbulidae Vigors, 1825} \\
\hline Brachygalba lugubris (Swainson, 1838) ${ }^{1 \mathrm{~A}}$ & ER & - & $\mathrm{F} 1$ & - \\
\hline Galbula ruficauda Cuvier, $1816^{1}$ & $\mathrm{C}$ & $\mathrm{C}$ & $\mathrm{F} 2$ & - \\
\hline \multicolumn{5}{|l|}{ Bucconidae Horsfield, 1821} \\
\hline Notharchus tectus (Boddaert, 1783) ${ }^{1 \mathrm{~A} *}$ & $\mathrm{R}$ & $\mathrm{R}$ & $\mathrm{F} 1$ & - \\
\hline Bucco tamatia Gmelin, $1788^{1 *}$ & $\mathrm{R}$ & I & $\mathrm{F} 1$ & ENA \\
\hline Nystalus chacuru (Vieillot, 1816) & I & - & $\mathrm{C} 1$ & - \\
\hline Nystalus maculatus (Gmelin, 1788) ${ }^{1 \mathrm{~A}}$ & I & I & $\mathrm{C} 2$ & - \\
\hline Monasa nigrifrons (Spix, 1824) ${ }^{1}$ & $\mathrm{C}$ & $\mathrm{C}$ & $\mathrm{F} 2$ & - \\
\hline Chelidoptera tenebrosa (Pallas, 1782) ${ }^{1}$ & $\mathrm{RC}$ & $\mathrm{RC}$ & $\mathrm{F} 2$ & - \\
\hline \multicolumn{5}{|l|}{ Ramphastidae Vigors, 1825} \\
\hline Ramphastos toco Statius Muller, $1776^{1}$ & $\mathrm{RC}$ & I & $\mathrm{C} 2$ & - \\
\hline Ramphastos vitellinus Lichtenstein, $1823^{1}$ & $\mathrm{RC}$ & $\mathrm{RC}$ & $\mathrm{F} 1$ & - \\
\hline Pteroglossus inscriptus Swainson, $1822^{1 *}$ & $\mathrm{R}$ & $\mathrm{RC}$ & $\mathrm{F} 1$ & ENA \\
\hline Pteroglossus aracari (Linnaeus, 1758) ${ }^{1}$ & $\mathrm{I}$ & $\mathrm{RC}$ & $\mathrm{F} 2$ & - \\
\hline Pteroglossus castanotis Gould, 1834 & $\mathrm{R}$ & - & $\mathrm{F} 2$ & - \\
\hline \multicolumn{5}{|l|}{ Picidae Leach, 1820} \\
\hline Picumnus albosquamatus d'Orbigny, $1840^{1}$ & I & I & $\mathrm{F} 2$ & - \\
\hline Melanerpes candidus $\left(\right.$ Otto, 1796) ${ }^{1}$ & $\mathrm{RC}$ & $\mathrm{RC}$ & $\mathrm{C} 2$ & - \\
\hline Melanerpes cruentatus (Boddaert, 1783) ${ }^{1}$ & I & $\mathrm{RC}$ & $\mathrm{F} 2$ & - \\
\hline Veniliornis affinis (Swainson, 1821) ${ }^{2}$ & - & - & - & - \\
\hline Veniliornis passerinus (Linnaeus, 1766) ${ }^{2}$ & - & - & - & - \\
\hline Piculus flavigula (Boddaert, 1783) ${ }^{1}$ & $\mathrm{R}$ & $\mathrm{RC}$ & $\mathrm{F} 1$ & - \\
\hline Piculus chrysochloros (Vieillot, 1818) ${ }^{1}$ & I & - & $\mathrm{F} 2$ & - \\
\hline Colaptes melanochloros $\left(\right.$ Gmelin, 1788) ${ }^{1}$ & - & $\mathrm{R}$ & $\mathrm{C} 2$ & - \\
\hline
\end{tabular}


Tabela 1. Continuação...

\begin{tabular}{|c|c|c|c|c|}
\hline Táxons/abundância relativa & $\begin{array}{c}\text { APA Ilha do Bananal/ } \\
\text { Cantão }\end{array}$ & $\begin{array}{c}\text { Parque Estadual } \\
\text { do Cantão }\end{array}$ & Habitat & Status \\
\hline Colaptes campestris (Vieillot, 1818) ${ }^{1 \mathrm{~A}}$ & $\mathrm{RC}$ & $\mathrm{R}$ & $\mathrm{C} 2$ & - \\
\hline Celeus elegans (Statius Muller, 1776)* & - & ER & $\mathrm{F} 1$ & ENA \\
\hline Celeus flavescens (Gmelin, 1788) ${ }^{1}$ & $\mathrm{RC}$ & $\mathrm{C}$ & $\mathrm{F} 2$ & - \\
\hline Celeus flavus (Statius Muller, 1776) ${ }^{1}$ & $\mathrm{R}$ & $\mathrm{R}$ & $\mathrm{F} 1$ & - \\
\hline Celeus torquatus (Boddaert, 1783) ${ }^{1 *}$ & - & ER & $\mathrm{F} 2$ & - \\
\hline Dryocopus lineatus (Linnaeus, 1766) ${ }^{1}$ & I & $\mathrm{RC}$ & $\mathrm{C} 2$ & - \\
\hline Campephilus rubricollis (Boddaert, 1783) ${ }^{1 *}$ & $\mathrm{R}$ & $\mathrm{R}$ & $\mathrm{F} 1$ & ENA \\
\hline Campephilus melanoleucos (Gmelin, 1788) ${ }^{1}$ & $\mathrm{R}$ & $\mathrm{R}$ & $\mathrm{F} 2$ & - \\
\hline \multicolumn{5}{|c|}{ Melanopareiidae Irestedt, Fjeldså, Johansson \& Ericson, 2002} \\
\hline Melanopareia torquata (Wied, 1831) & $\mathrm{R}$ & - & $\mathrm{C} 1$ & ENC \\
\hline \multicolumn{5}{|l|}{ Thamnophilidae Swainson, 1824} \\
\hline Taraba major (Vieillot, 1816) ${ }^{1}$ & I & $\mathrm{R}$ & $\mathrm{F} 2$ & - \\
\hline Sakesphorus luctuosus (Lichtenstein, 1823) ${ }^{1 *}$ & $\mathrm{RC}$ & $\mathrm{C}$ & $\mathrm{F} 1$ & ENA \\
\hline Thamnophilus doliatus (Linnaeus, 1764) ${ }^{1}$ & - & $\mathrm{C}$ & $\mathrm{F} 2$ & - \\
\hline Thamnophilus pelzelni Hellmayr, $1924^{1 \mathrm{~A}}$ & $\mathrm{R}$ & I & $\mathrm{C} 2$ & - \\
\hline Thamnophilus amazonicus Sclater, $1858^{1 *}$ & - & I & $\mathrm{F} 1$ & ENA \\
\hline Dysithamnus mentalis (Temminck, 1823) ${ }^{1}$ & $\mathrm{RC}$ & I & F1 & - \\
\hline Myrmotherula multostriata Sclater, $1858^{1}$ & - & I & $\mathrm{F} 1$ & \\
\hline Myrmotherula axillaris (Vieillot, 1817) ${ }^{1}$ & - & I & $\mathrm{F} 1$ & - \\
\hline Herpsilochmus atricapillus Pelzeln, $1868^{1}$ & $\mathrm{C}$ & $\mathrm{RC}$ & $\mathrm{F} 1$ & - \\
\hline Herpsilochmus longirostris Pelzeln, $1868^{1}$ & - & $\mathrm{R}$ & $\mathrm{F} 1$ & ENC \\
\hline Formicivora grisea (Boddaert, 1783) ${ }^{1}$ & $\mathrm{C}$ & $\mathrm{C}$ & $\mathrm{F} 2$ & - \\
\hline Formicivora rufa $\left(\right.$ Wied, 1831) ${ }^{1}$ & I & I & $\mathrm{C} 2$ & - \\
\hline Cercomacra ferdinandi Snethlage, $1928^{1}$ & I & $\mathrm{RC}$ & $\mathrm{F} 1$ & $\mathrm{VU}^{\mathrm{A}, \mathrm{B}}, \mathrm{ENC}$ \\
\hline Hypocnemoides maculicauda $(\text { Pelzeln, } 1868)^{1 *}$ & $\mathrm{RC}$ & $\mathrm{C}$ & $\mathrm{F} 1$ & ENA \\
\hline \multicolumn{5}{|l|}{ Dendrocolaptidae Gray, 1840} \\
\hline Dendrocincla fuliginosa $\left(\right.$ Vieillot, 1818) ${ }^{1 *}$ & $\mathrm{R}$ & - & $\mathrm{F} 1$ & - \\
\hline Sittasomus griseicapillus (Vieillot, 1818) ${ }^{1}$ & I & I & $\mathrm{F} 1$ & - \\
\hline Nasica longirostris (Vieillot, 1818) ${ }^{1 *}$ & - & $\mathrm{RC}$ & $\mathrm{F} 2$ & ENA \\
\hline Dendrocolaptes certhia (Boddaert, 1783)* & I & I & $\mathrm{F} 1$ & ENA \\
\hline Xiphorhynchus picus (Gmelin, 1788) ${ }^{1}$ & I & $\mathrm{RC}$ & $\mathrm{F} 2$ & - \\
\hline Xiphorhynchus obsoletus (Lichtenstein, 1820) ${ }^{2}$ & - & - & - & - \\
\hline Xiphorhynchus guttatus (Lichtenstein, 1820) ${ }^{1}$ & $\mathrm{R}$ & $\mathrm{R}$ & $\mathrm{F} 1$ & ENA \\
\hline Lepidocolaptes angustirostris (Vieillot, 1818) ${ }^{1}$ & I & - & $\mathrm{C} 2$ & - \\
\hline \multicolumn{5}{|l|}{ Furnariidae Gray, 1840} \\
\hline Furnarius figulus (Lichtenstein, 1823) ${ }^{1}$ & - & $\mathrm{RC}$ & $\mathrm{C} 2$ & - \\
\hline Furnarius leucopus Swainson, $1838^{1}$ & - & $\mathrm{RC}$ & $\mathrm{C} 2$ & - \\
\hline Furnarius rufus (Gmelin, 1788) & $\mathrm{R}$ & - & $\mathrm{C} 2$ & - \\
\hline Synallaxis albescens Temminck, $1823^{1}$ & $\mathrm{R}$ & - & $\mathrm{C} 1$ & - \\
\hline Synallaxis simoni Hellmayr, $1907^{1}$ & - & ER & $\mathrm{C} 1$ & $\mathrm{VU}^{\mathrm{A}, \mathrm{B}}, \mathrm{ENC}$ \\
\hline Cranioleuca vulpina (Pelzeln, 1856) ${ }^{1}$ & I & $\mathrm{RC}$ & $\mathrm{F} 1$ & - \\
\hline Certhiaxis cinnamomeus (Gmelin, 1788) ${ }^{1}$ & $\mathrm{RC}$ & $\mathrm{C}$ & A & - \\
\hline Certhiaxis sp.1 & - & $\mathrm{RC}$ & A & - \\
\hline Phacellodomus ruber (Vieillot, 1817) ${ }^{1}$ & $\mathrm{R}$ & $\mathrm{RC}$ & $\mathrm{C} 2$ & - \\
\hline Xenops minutus (Sparrman, 1788) ${ }^{1}$ & $\mathrm{R}$ & $\mathrm{R}$ & F1 & - \\
\hline Xenops rutilans Temminck, $1821^{2}$ & - & - & - & - \\
\hline \multicolumn{5}{|l|}{ Tyrannidae Vigors, 1825} \\
\hline Leptopogon amaurocephalus Tschudi, $1846^{1}$ & $\mathrm{R}$ & - & $\mathrm{F} 1$ & - \\
\hline Hemitriccus minor (Snethlage, 1907) ${ }^{2}$ & - & - & - & - \\
\hline Corythopis torquatus (Tschudi, 1844)* & ER & - & $\mathrm{F} 1$ & ENA \\
\hline
\end{tabular}


Tabela 1. Continuação...

\begin{tabular}{|c|c|c|c|c|}
\hline Táxons/abundância relativa & $\begin{array}{l}\text { APA Ilha do Bananal/ } \\
\text { Cantão }\end{array}$ & $\begin{array}{c}\text { Parque Estadual } \\
\text { do Cantão }\end{array}$ & Habitat & Status \\
\hline Hemitriccus striaticollis (Lafresnaye, 1853) ${ }^{1 *}$ & I & $\mathrm{I}$ & $\mathrm{F} 1$ & - \\
\hline Hemitriccus minimus (Todd, 1925) ${ }^{1 *}$ & - & I & $\mathrm{F} 1$ & ENA \\
\hline Poecilotriccus fumifrons (Hartlaub, 1853) ${ }^{1}$ & - & I & F1 & - \\
\hline Poecilotriccus latirostris (Pelzeln, 1868) ${ }^{1}$ & - & I & F1 & - \\
\hline Todirostrum maculatum (Desmarest, 1806) ${ }^{1}$ & - & $\mathrm{C}$ & F1 & ENA \\
\hline Todirostrum cinereum (Linnaeus, 1766) ${ }^{1}$ & $\mathrm{R}$ & - & $\mathrm{F} 2$ & - \\
\hline Tyrannulus elatus (Latham, 1790) ${ }^{1}$ & $\mathrm{R}$ & - & $\mathrm{C} 2$ & - \\
\hline Myiopagis gaimardii (d'Orbigny, 1839) ${ }^{1}$ & I & $\mathrm{R}$ & F1 & - \\
\hline Myiopagis viridicata (Vieillot, 1817) ${ }^{1}$ & I & $\mathrm{RC}$ & $\mathrm{F} 1$ & - \\
\hline Elaenia flavogaster (Thunberg, 1822) ${ }^{1}$ & I & I & $\mathrm{C} 2$ & - \\
\hline Elaenia spectabilis Pelzeln, $1868^{1}$ & - & I & $\mathrm{C} 2$ & - \\
\hline Elaenia parvirostris Pelzeln, 1868 & I & - & $\mathrm{C} 1$ & - \\
\hline Elaenia cristata Pelzeln, $1868^{1 \mathrm{~A}}$ & I & I & $\mathrm{C} 1$ & - \\
\hline Elaenia chiriquensis Lawrence, 1865 & I & $\mathrm{I}$ & $\mathrm{C} 1$ & - \\
\hline Ornithion inerme Hartlaub, $1853 *$ & $\mathrm{R}$ & $\mathrm{R}$ & $\mathrm{F} 1$ & - \\
\hline Camptostoma obsoletum (Temminck, 1824) ${ }^{1}$ & I & $\mathrm{RC}$ & $\mathrm{F} 2$ & - \\
\hline Suiriri suiriri (Vieillot, 1818) & $\mathrm{R}$ & - & $\mathrm{C} 2$ & - \\
\hline Serpophaga hypoleuca Sclater \& Salvin, $1866^{1}$ & - & I & $\mathrm{C} 2$ & ENA \\
\hline Phaeomyias murina $(\text { Spix, } 1825)^{1}$ & $\mathrm{R}$ & - & $\mathrm{F} 2$ & - \\
\hline Euscarthmus meloryphus Wied, $1831^{2}$ & - & - & - & - \\
\hline Inezia subflava (Sclater \& Salvin, 1873) ${ }^{1}$ & - & $\mathrm{RC}$ & $\mathrm{F} 1$ & - \\
\hline Myiornis ecaudatus (d'Orbigny \& Lafresnaye, 1837) & - & ER & $\mathrm{F} 2$ & - \\
\hline Tolmomyias sulphurescens (Spix, 1825) ${ }^{1}$ & $\mathrm{R}$ & $\mathrm{RC}$ & F1 & - \\
\hline Tolmomyias assimilis (Pelzeln, 1868) ${ }^{2}$ & - & - & - & - \\
\hline Tolmomyias poliocephalus (Taczanowski, 1884)² & - & - & - & - \\
\hline Tolmomyias flaviventris (Wied, 1831) ${ }^{1}$ & I & - & $\mathrm{F} 1$ & - \\
\hline Platyrinchus mystaceus Vieillot, 1818 & $\mathrm{R}$ & $\mathrm{R}$ & $\mathrm{F} 1$ & - \\
\hline Platyrinchus platyrhynchos (Gmelin, 1788)2 & - & - & - & - \\
\hline Myiophobus fasciatus (Statius Muller, 1776) ${ }^{1}$ & $\mathrm{R}$ & - & $\mathrm{C} 2$ & - \\
\hline Myiobius atricaudus Lawrence, $1863^{1}$ & ER & - & F1 & - \\
\hline Terenotriccus erythrurus (Cabanis, 1847)* & $\mathrm{R}$ & - & $\mathrm{F} 1$ & - \\
\hline Lathrotriccus euleri (Cabanis, 1868) ${ }^{1}$ & I & I & $\mathrm{F} 1$ & - \\
\hline Cnemotriccus fuscatus (Wied, 1831) ${ }^{1}$ & I & I & F1 & - \\
\hline Pyrocephalus rubinus (Boddaert, 1783) & - & - & - & - \\
\hline Knipolegus poecilocercus (Pelzeln, 1868) ${ }^{1}$ & - & ER & F1 & ENA \\
\hline Knipolegus orenocensis Berlepsch, $1864^{1 *}$ & - & I & $\mathrm{F} 1$ & ENA \\
\hline Satrapa icterophrys (Vieillot, 1818) ${ }^{1}$ & - & ER & $\mathrm{C} 2$ & - \\
\hline Xolmis cinereus (Vieillot, 1816) & $\mathrm{RC}$ & - & $\mathrm{C} 1$ & - \\
\hline Fluvicola albiventer (Spix, 1825) ${ }^{1}$ & $\mathrm{RC}$ & I & A & - \\
\hline Colonia colonus (Vieillot, 1818) ${ }^{2}$ & - & - & - & - \\
\hline Arundinicola leucocephala (Linnaeus, 1764) ${ }^{1}$ & $\mathrm{RC}$ & I & A & - \\
\hline Legatus leucophaius (Vieillot, 1818) ${ }^{1}$ & $\mathrm{RC}$ & $\mathrm{R}$ & $\mathrm{F} 2$ & - \\
\hline Myiozetetes cayanensis (Linnaeus, 1766) ${ }^{1}$ & $\mathrm{RC}$ & $\mathrm{C}$ & $\mathrm{F} 2$ & - \\
\hline Myiozetetes similis (Spix, 1825) ${ }^{1}$ & I & - & $\mathrm{F} 2$ & - \\
\hline Pitangus sulphuratus (Linnaeus, 1766) ${ }^{1}$ & $\mathrm{C}$ & $\mathrm{C}$ & $\mathrm{C} 2$ & - \\
\hline Philohydor lictor (Lichtenstein, 1823) ${ }^{1}$ & $\mathrm{R}$ & $\mathrm{RC}$ & $\mathrm{C} 2$ & - \\
\hline Myiodynastes maculatus (Statius Muller, 1776) ${ }^{1 \mathrm{~A}}$ & I & $\mathrm{R}$ & $\mathrm{F} 2$ & - \\
\hline Megarynchus pitangua (Linnaeus, 1766) ${ }^{1}$ & $\mathrm{RC}$ & I & $\mathrm{F} 2$ & - \\
\hline Empidonomus varius (Vieillot, 1818) & - & $\mathrm{R}$ & $\mathrm{F} 2$ & - \\
\hline
\end{tabular}


Tabela 1. Continuação...

\begin{tabular}{|c|c|c|c|c|}
\hline Táxons/abundância relativa & $\begin{array}{l}\text { APA Ilha do Bananal/ } \\
\text { Cantão }\end{array}$ & $\begin{array}{c}\text { Parque Estadual } \\
\text { do Cantão }\end{array}$ & Habitat & Status \\
\hline $\begin{array}{l}\text { Griseotyrannus aurantioatrocristatus } \\
\text { (d'Orbigny \& Lafresnaye, 1837) }{ }^{1}\end{array}$ & $\mathrm{R}$ & - & $\mathrm{C} 2$ & - \\
\hline Tyrannus albogularis Burmeister, $1856^{1}$ & I & - & $\mathrm{C} 1$ & - \\
\hline Tyrannus melancholicus Vieillot, $1819^{1}$ & $\mathrm{C}$ & $\mathrm{RC}$ & $\mathrm{C} 2$ & - \\
\hline Tyrannus savana Vieillot, $1808^{1}$ & $\mathrm{RC}$ & $\mathrm{R}$ & $\mathrm{C} 1$ & - \\
\hline Rhytipterna simplex (Lichtenstein, 1823) ${ }^{1}$ & $\mathrm{R}$ & - & F1 & - \\
\hline Sirystes sibilator (Vieillot, 1818) & $\mathrm{R}$ & - & F1 & - \\
\hline Casiornis fuscus Sclater \& Salvin, $1873^{2}$ & - & - & - & - \\
\hline Casiornis rufus (Vieillot, 1816) ${ }^{2 \mathrm{~A}}$ & - & - & - & - \\
\hline Myiarchus tuberculifer (d'Orbigny \& Lafresnaye, 1837) ${ }^{1 *}$ & $\mathrm{R}$ & $\mathrm{R}$ & $\mathrm{F} 2$ & - \\
\hline Myiarchus swainsoni Cabanis \& Heine, 1859 & I & - & F2 & - \\
\hline Myiarchus ferox (Gmelin, 1789) ${ }^{1}$ & I & $\mathrm{RC}$ & F2 & - \\
\hline Myiarchus tyrannulus (Statius Muller, 1776) ${ }^{1 \mathrm{~A}}$ & - & $\mathrm{R}$ & $\mathrm{F} 2$ & - \\
\hline Ramphotrigon ruficauda $(\text { Spix, 1825) })^{2}$ & - & - & & - \\
\hline Attila cinnamomeus (Gmelin, 1789) ${ }^{1}$ & - & $\mathrm{RC}$ & $\mathrm{F} 1$ & ENA \\
\hline \multicolumn{5}{|l|}{ Cotingidae Bonaparte, 1849} \\
\hline Lipaugus vociferans (Wied, 1820$)^{1 *}$ & $\mathrm{C}$ & - & F1 & - \\
\hline Gymnoderus foetidus (Linnaeus, 1758) ${ }^{1 *}$ & I & $\mathrm{RC}$ & $\mathrm{F} 1$ & ENA \\
\hline Querula purpurata (Statius Muller, 1776) ${ }^{1 *}$ & $\mathrm{RC}$ & $\mathrm{RC}$ & $\mathrm{F} 1$ & - \\
\hline \multicolumn{5}{|l|}{ Pipridae Rafinesque, 1815} \\
\hline Neopelma pallescens (Lafresnaye, 1853) & I & - & $\mathrm{F} 1$ & - \\
\hline Tyranneutes stolzmanni (Hellmayr, 1906) $)^{1 *}$ & I & $\mathrm{C}$ & $\mathrm{F} 1$ & ENA \\
\hline Machaeropterus pyrocephalus (Sclater, 1852)* & I & ER & $\mathrm{F} 1$ & ENA \\
\hline Manacus manacus (Linnaeus, 1766) ${ }^{1}$ & I & $\mathrm{C}$ & $\mathrm{F} 1$ & - \\
\hline Chiroxiphia pareola (Linnaeus, 1766)* & $\mathrm{R}$ & & $\mathrm{F} 1$ & \\
\hline Xenopipo atronitens Cabanis, 1847 & - & ER & F1 & ENA \\
\hline Heterocercus linteatus (Strickland, 1850)* & - & ER & $\mathrm{F} 1$ & ENA \\
\hline Pipra fasciicauda Hellmayr, $1906^{1}$ & $\mathrm{RC}$ & $\mathrm{C}$ & $\mathrm{F} 1$ & - \\
\hline Pipra rubrocapilla Temminck, $1821^{*}$ & $\mathrm{RC}$ & - & $\mathrm{F} 1$ & - \\
\hline \multicolumn{5}{|l|}{ Tityridae Gray, 1840} \\
\hline Tityra inquisitor (Lichtenstein, 1823) $)^{1}$ & - & $\mathrm{R}$ & $\mathrm{F} 1$ & - \\
\hline Tityra cayana (Linnaeus, 1766) & I & $\mathrm{C}$ & $\mathrm{F} 1$ & - \\
\hline Tityra semifasciata (Spix, 1825) $)^{1 *}$ & I & $\mathrm{R}$ & $\mathrm{F} 1$ & - \\
\hline Pachyramphus polychopterus (Vieillot, 1818) & $\mathrm{R}$ & - & $\mathrm{F} 2$ & - \\
\hline Xenopsaris albinucha (Burmeister, 1869) ${ }^{2}$ & - & - & - & - \\
\hline \multicolumn{5}{|l|}{ Vireonidae Swainson, 1837} \\
\hline Cyclarhis gujanensis (Gmelin, 1789) ${ }^{1 *}$ & $\mathrm{RC}$ & - & $\mathrm{F} 2$ & - \\
\hline${\text { Vireo olivaceus (Linnaeus, } 1766)^{1}}$ & $\mathrm{RC}$ & I & F2 & - \\
\hline Hylophilus semicinereus Sclater \& Salvin, $1867^{1 *}$ & - & I & $\mathrm{F} 1$ & ENA \\
\hline Hylophilus pectoralis Sclater, $1866^{1 *}$ & I & $\mathrm{RC}$ & $\mathrm{F} 1$ & ENA \\
\hline \multicolumn{5}{|l|}{ Corvidae Leach, 1820} \\
\hline Cyanocorax cristatellus (Temminck, 1823$)^{2}$ & - & - & - & - \\
\hline Cyanocorax cyanopogon (Wied, 1821) & $\mathrm{RC}$ & $\mathrm{RC}$ & F2 & - \\
\hline \multicolumn{5}{|l|}{ Hirundinidae Rafinesque, 1815} \\
\hline Tachycineta albiventer (Boddaert, 1783) ${ }^{1}$ & $\mathrm{RC}$ & $\mathrm{C}$ & A & - \\
\hline Progne tapera $(\text { Vieillot, } 1817)^{1}$ & $\mathrm{RC}$ & $\mathrm{RC}$ & $\mathrm{C} 2$ & - \\
\hline Progne subis (Linnaeus, 1758) & $\mathrm{C}$ & $\mathrm{C}$ & $\mathrm{C} 1$ & $\mathrm{VN}$ \\
\hline Progne chalybea (Gmelin, 1789) $)^{1}$ & $\mathrm{RC}$ & $\mathrm{RC}$ & $\mathrm{C} 2$ & - \\
\hline Stelgidopteryx ruficollis (Vieillot, 1817) & $\mathrm{RC}$ & $\mathrm{C}$ & $\mathrm{C} 2$ & - \\
\hline
\end{tabular}


Tabela 1. Continuação...

\begin{tabular}{|c|c|c|c|c|}
\hline Táxons/abundância relativa & $\begin{array}{c}\text { APA Ilha do Bananal/ } \\
\text { Cantão }\end{array}$ & $\begin{array}{c}\text { Parque Estadual } \\
\text { do Cantão }\end{array}$ & Habitat & Status \\
\hline Riparia riparia (Linnaeus, 1758) & - & - & - & - \\
\hline Hirundo rustica Linnaeus, $1758^{1}$ & - & $\mathrm{R}$ & $\mathrm{C} 1$ & VN \\
\hline \multicolumn{5}{|l|}{ Troglodytidae Swainson, 1831} \\
\hline Troglodytes musculus Naumann, $1823^{1}$ & $\mathrm{RC}$ & $\mathrm{R}$ & $\mathrm{C} 2$ & - \\
\hline Pheugopedius genibarbis (Swainson, 1838) ${ }^{1}$ & $\mathrm{RC}$ & $\mathrm{RC}$ & $\mathrm{F} 1$ & - \\
\hline Cantorchilus leucotis (Lafresnaye, 1845) ${ }^{1}$ & $\mathrm{C}$ & $\mathrm{C}$ & $\mathrm{F} 1$ & - \\
\hline \multicolumn{5}{|l|}{ Donacobiidae Aleixo \& Pacheco, 2006} \\
\hline Donacobius atricapilla (Linnaeus, 1766) ${ }^{1}$ & I & $\mathrm{RC}$ & A & - \\
\hline \multicolumn{5}{|l|}{ Polioptilidae Baird, 1858} \\
\hline Polioptila dumicola (Vieillot, 1817) ${ }^{1}$ & I & $\mathrm{RC}$ & $\mathrm{F} 2$ & - \\
\hline \multicolumn{5}{|l|}{ Turdidae Rafinesque, 1815} \\
\hline Turdus leucomelas Vieillot, $1818^{1 \mathrm{~A}}$ & $\mathrm{C}$ & $\mathrm{C}$ & $\mathrm{F} 2$ & - \\
\hline Turdus amaurochalinus Cabanis, $1850^{1}$ & $\mathrm{R}$ & - & $\mathrm{C} 2$ & - \\
\hline Turdus fumigatus Lichtenstein, $1823^{1 *}$ & - & ER & $\mathrm{F} 1$ & - \\
\hline Turdus albicollis Vieillot, 1818 & $\mathrm{R}$ & - & F1 & - \\
\hline \multicolumn{5}{|l|}{ Mimidae Bonaparte, 1853} \\
\hline Mimus saturninus (Lichtenstein, 1823) & $\mathrm{RC}$ & I & $\mathrm{C} 1$ & - \\
\hline \multicolumn{5}{|l|}{ Motacillidae Horsfield, 1821} \\
\hline Anthus lutescens Pucheran, $1855^{1}$ & - & I & $\mathrm{C} 1$ & - \\
\hline \multicolumn{5}{|l|}{ Coerebidae d'Orbigny \& Lafresnaye, 1838} \\
\hline Coereba flaveola (Linnaeus, 1758) ${ }^{1}$ & $\mathrm{RC}$ & $\mathrm{C}$ & $\mathrm{F} 2$ & - \\
\hline \multicolumn{5}{|l|}{ Thraupidae Cabanis, 1847} \\
\hline Schistochlamys melanopis (Latham, 1790) & $\mathrm{R}$ & $\mathrm{R}$ & $\mathrm{C} 2$ & - \\
\hline Schistochlamys ruficapillus (Vieillot, 1817) & $\mathrm{R}$ & ER & $\mathrm{C} 2$ & - \\
\hline Cissopis leverianus $(\mathrm{Gmelin}, 1788)^{1 \mathrm{~A}}$ & $\mathrm{R}$ & - & $\mathrm{F} 1$ & - \\
\hline Compsothraupis loricata (Lichtenstein, 1819) & - & ER & $\mathrm{C} 2$ & - \\
\hline Nemosia pileata (Boddaert, 1783) & I & $\mathrm{R}$ & $\mathrm{F} 2$ & - \\
\hline Thlypopsis sordida (d'Orbigny \& Lafresnaye, 1837) ${ }^{1}$ & - & I & $\mathrm{F} 2$ & - \\
\hline Cypsnagra hirundinacea (Lesson, 1831) & $\mathrm{R}$ & - & $\mathrm{C} 1$ & - \\
\hline Piranga flava (Vieillot, 1822) & - & ER & $\mathrm{C} 2$ & - \\
\hline Eucometis penicillata $\left(\right.$ Spix, 1825) ${ }^{1}$ & - & $\mathrm{R}$ & F1 & - \\
\hline Tachyphonus cristatus (Linnaeus, 1766) ${ }^{1}$ & $\mathrm{RC}$ & I & $\mathrm{F} 1$ & - \\
\hline Tachyphonus luctuosus d'Orbigny \& Lafresnaye, $1837^{1 *}$ & $\mathrm{R}$ & I & $\mathrm{F} 1$ & - \\
\hline Tachyphonus rufus (Boddaert, 1783) ${ }^{1}$ & I & I & $\mathrm{F} 2$ & - \\
\hline Ramphocelus carbo (Pallas, 1764) ${ }^{1}$ & $\mathrm{C}$ & $\mathrm{C}$ & $\mathrm{F} 2$ & - \\
\hline Thraupis sayaca (Linnaeus, 1766) ${ }^{1}$ & $\mathrm{C}$ & $\mathrm{R}$ & $\mathrm{C} 2$ & - \\
\hline Thraupis palmarum $(\text { Wied, } 1823)^{1}$ & $\mathrm{C}$ & $\mathrm{RC}$ & $\mathrm{F} 2$ & - \\
\hline Tangara cayana (Linnaeus, 1766) & $\mathrm{RC}$ & $\mathrm{R}$ & $\mathrm{C} 2$ & - \\
\hline Tersina viridis (Illiger, 1811) ${ }^{1}$ & $\mathrm{R}$ & $\mathrm{R}$ & $\mathrm{F} 2$ & - \\
\hline Dacnis cayana (Linnaeus, 1766) ${ }^{1}$ & $\mathrm{RC}$ & I & $\mathrm{F} 2$ & - \\
\hline Cyanerpes cyaneus (Linnaeus, 1766) & I & - & $\mathrm{F} 1$ & - \\
\hline Hemithraupis guira (Linnaeus, 1766) ${ }^{1}$ & I & - & $\mathrm{C} 2$ & - \\
\hline Conirostrum speciosum (Temminck, 1824) & - & $\mathrm{I}$ & $\mathrm{F} 2$ & - \\
\hline \multicolumn{5}{|l|}{ Emberizidae Vigors, 1825} \\
\hline Ammodramus humeralis $(\text { Bosc, } 1792)^{1 \mathrm{~A}}$ & $\mathrm{RC}$ & $\mathrm{RC}$ & $\mathrm{C} 1$ & - \\
\hline Ammodramus aurifrons $\left(\right.$ Spix, 1825) ${ }^{1}$ & - & $\mathrm{C}$ & A & - \\
\hline Sicalis columbiana Cabanis, $1851^{1}$ & $\mathrm{RC}$ & $\mathrm{RC}$ & A & - \\
\hline Emberizoides herbicola (Vieillot, 1817) ${ }^{1}$ & I & I & $\mathrm{C} 1$ & - \\
\hline Volatinia jacarina (Linnaeus, 1766$)^{1}$ & $\mathrm{RC}$ & $\mathrm{RC}$ & $\mathrm{C} 1$ & - \\
\hline
\end{tabular}


Tabela 1. Continuação...

\begin{tabular}{|c|c|c|c|c|}
\hline Táxons/abundância relativa & $\begin{array}{c}\text { APA Ilha do Bananal/ } \\
\text { Cantão }\end{array}$ & $\begin{array}{l}\text { Parque Estadual } \\
\text { do Cantão }\end{array}$ & Habitat & Status \\
\hline Sporophila schistacea (Lawrence, 1862) ${ }^{1 *}$ & $\mathrm{R}$ & - & $\mathrm{C} 1$ & - \\
\hline Sporophila plumbea $(\text { Wied, } 1830)^{1 \mathrm{~A}}$ & I & I & $\mathrm{C} 1$ & - \\
\hline Sporophila collaris (Boddaert, 1783) ${ }^{1}$ & $\mathrm{R}$ & $\mathrm{R}$ & A & - \\
\hline Sporophila lineola (Linnaeus, 1758$)^{1}$ & I & I & $\mathrm{C} 1$ & - \\
\hline Sporophila nigricollis (Vieillot, 1823) ${ }^{1}$ & $\mathrm{R}$ & $\mathrm{R}$ & $\mathrm{C} 2$ & - \\
\hline Sporophila caerulescens $\left(\right.$ Vieillot, 1823) ${ }^{1}$ & I & I & $\mathrm{C} 2$ & - \\
\hline Sporophila leucoptera (Vieillot, 1817)2 & - & - & - & - \\
\hline Sporophila bouvreuil (Statius Muller, 1776) ${ }^{1}$ & $\mathrm{R}$ & - & $\mathrm{C} 2$ & - \\
\hline Sporophila castaneiventris Cabanis, $1849^{1}$ & & I & $\mathrm{C} 2$ & ENA \\
\hline Sporophila angolensis (Linnaeus, 1766) ${ }^{1}$ & I & I & $\mathrm{C} 2$ & - \\
\hline Arremon taciturnus (Hermann, 1783) ${ }^{1}$ & I & $\mathrm{RC}$ & F1 & - \\
\hline Charitospiza eucosma Oberholser, 1905 & - & - & $\mathrm{C} 1$ & $\mathrm{NT}^{\mathrm{B}}, \mathrm{ENC}$ \\
\hline Coryphospingus pileatus (Wied, 1821) & I & I & $\mathrm{C} 2$ & - \\
\hline Paroaria gularis (Linnaeus, 1766) ${ }^{1}$ & $\mathrm{R}$ & I & $\mathrm{F} 2$ & - \\
\hline Paroaria baeri Hellmayr, 1907 & - & ER & $\mathrm{F} 2$ & ENC \\
\hline \multicolumn{5}{|l|}{ Cardinalidae Ridgway, 1901} \\
\hline Saltator maximus (Statius Muller, 1776) ${ }^{1}$ & $\mathrm{RC}$ & $\mathrm{R}$ & $\mathrm{F} 2$ & - \\
\hline Saltator coerulescens Vieillot, $1817^{1}$ & - & $\mathrm{RC}$ & $\mathrm{F} 2$ & - \\
\hline Saltator atricollis Vieillot, 1817 & I & - & $\mathrm{C} 1$ & ENC \\
\hline Cyanocompsa cyanoides (Lafresnaye, 1847)2 & - & - & - & - \\
\hline \multicolumn{5}{|c|}{ Parulidae Wetmore, Friedmann, Lincoln, Miller, Peters, van Rossem, van Tyne \& Zimmer 1947} \\
\hline Basileuterus culicivorus (Deppe, 1830) & $\mathrm{RC}$ & I & F1 & - \\
\hline Basileuterus flaveolus (Baird, 1865) ${ }^{1}$ & $\mathrm{RC}$ & $\mathrm{R}$ & F1 & - \\
\hline Granatellus pelzelni Sclater, $1865^{1}$ & - & $\mathrm{R}$ & $\mathrm{F} 1$ & ENA \\
\hline \multicolumn{5}{|l|}{ Icteridae Vigors, 1825} \\
\hline Psarocolius decumanus (Pallas, 1769) ${ }^{1}$ & I & I & $\mathrm{F} 2$ & - \\
\hline Cacicus solitarius (Vieillot, 1816) ${ }^{1}$ & $\mathrm{RC}$ & $\mathrm{C}$ & $\mathrm{F} 2$ & - \\
\hline Cacicus cela (Linnaeus, 1758) ${ }^{1 *}$ & $\mathrm{C}$ & $\mathrm{C}$ & $\mathrm{F} 2$ & - \\
\hline 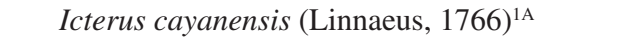 & $\mathrm{R}$ & $\mathrm{R}$ & $\mathrm{F} 2$ & - \\
\hline Icterus jamacaii (Gmelin, 1788) ${ }^{1}$ & - & $\mathrm{R}$ & $\mathrm{F} 2$ & - \\
\hline Gnorimopsar chopi (Vieillot, 1819) ${ }^{1 \mathrm{~A}}$ & $\mathrm{C}$ & I & $\mathrm{C} 2$ & - \\
\hline Molothrus oryzivorus $(\mathrm{Gmelin}, 1788)^{1}$ & - & $\mathrm{R}$ & $\mathrm{C} 2$ & - \\
\hline Molothrus bonariensis (Gmelin, 1789) ${ }^{1}$ & $\mathrm{R}$ & $\mathrm{R}$ & $\mathrm{C} 2$ & - \\
\hline Sturnella militaris (Linnaeus, 1758) & - & ER & $\mathrm{C} 1$ & - \\
\hline \multicolumn{5}{|l|}{ Fringillidae Leach, 1820} \\
\hline Euphonia chlorotica (Linnaeus, 1766) ${ }^{1}$ & $\mathrm{RC}$ & $\mathrm{RC}$ & $\mathrm{C} 2$ & - \\
\hline Euphonia violacea (Linnaeus, 1758) ${ }^{1}$ & $\mathrm{RC}$ & $\mathrm{RC}$ & $\mathrm{F} 2$ & - \\
\hline \multicolumn{5}{|l|}{ Passeridae Rafinesque, 1815} \\
\hline Passer domesticus (Linnaeus, 1758) ${ }^{1}$ & $\mathrm{RC}$ & - & $\mathrm{C} 1$ & - \\
\hline
\end{tabular}

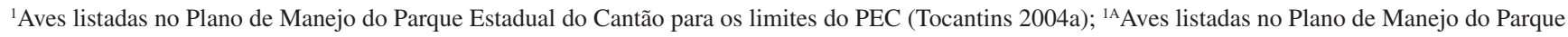
Estadual do Cantão fora dos limites do PEC (Tocantins 2004a); ${ }^{2}$ Aves listadas no Plano de Manejo do Parque Estadual do Cantão para os limites do PEC (Tocantins 2004a) não registradas neste estudo. ${ }^{2 \mathrm{~A}}$ Aves listadas no Plano de Manejo do Parque Estadual do Cantão fora dos limites do PEC (Tocantins 2004a) não registradas neste estudo (estas espécies não foram consideradas para a abundância relativa e preferência de hábitat); ${ }^{\mathrm{A} E s p e ́ c i e s ~ A m e a c ̧ a d a s ~ s e g-~}$

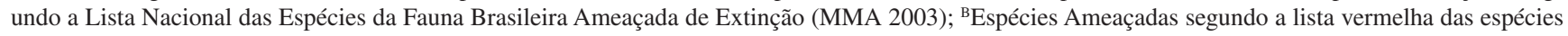
ameaçadas globalmente da IUCN (2006); *Espécies encontradas no Cerrado com centro de distribuição na Amazônia segundo Silva (1996); nomenclatura segundo o Comitê Brasileiro de Registros Ornitológicos (2007). Abundância relativa: Comum (C): espécie registrada entre 100\% e 75\% das visitas à campo; Relativamente Comum (RC): espécie registrada entre 74\% e 50\% das visitas à campo; Incomuns (I): espécie registrada entre 49\% e 25\% das visitas à campo; Raras (R): Espécie registrada entre $24 \%$ e $6 \%$ das visitas à campo; Extremamente Raras (ER): espécie registrada em 1 ou 2 ocasiões. Habitat: F1 - Estritamente Florestal; F2 - Florestal com utilização de área campestre ou aberta; C1 - Estritamente campestre; C2 - Campestre com utilização de área florestada; A - Ambiente aquático (rios, lagos, margem, praias). Endemismos: ENC - Espécies de aves endêmicas do Cerrado (Silva 1997, Silva e Santos 2005); ENA - Espécies de aves endêmicas da Amazônia, segundo Stotz et al. (1996). Nível de ameaça: NT - Quase ameaçada; VU - Vulnerável à extinção. Status migratório: VN - Visitante do Hemisfério Norte. 
um fornecido por Tocantins (2004a) e outro por este estudo. A espécie encontra-se na categoria de quase-ameaçada principalmente em função da degradação e perda do seu habitat natural e a caça (IUCN 2006). Entretanto, na área de estudo da APA e no Estado do Tocantins, esta espécie é comum, sendo avistada com freqüência tanto em áreas naturais do Cerrado como em áreas cultivadas e pastagens. Não há registro de pressão de caça localmente.

Neochen jubata (Spix, 1825) - considerada quase-ameaçada em função da forte pressão de caça e destruição dos rios para construção de complexos hidroelétricos (IUCN 2006), o pato-corredor é uma espécie relativamente comum no Parque Estadual do Cantão. É regularmente avistada em pequenos bandos de quatro a dez indivíduos, principalmente nos meses centrais do ano quando, em função da estiagem, se formam bancos de areia nas margens do rio do Côco, Javaés e Araguaia. De acordo com Birdlife (2006), as maiores populações desta espécie se encontram no norte da Bolívia e na Ilha do Bananal. Entretanto, em função da degradação dos ambientes naturais na Ilha do Bananal, o Parque Estadual do Cantão parece ser uma das principais áreas protegidas onde esta espécie ocorre no Brasil.

Penelope ochrogaster Pelzeln, 1870 - endêmica do Cerrado (Silva 1995, 1997) e globalmente ameaçada de extinção (MMA 2003, Machado et al. 2005, IUCN 2006), o jacu-de-barriga-vermelha apresenta alta densidade populacional na RPPN SESC-Pantanal (Antas 2004). Segundo Olmos (2003) a região norte do Pantanal do Mato Grosso parece ser a área que comporta as maiores populações desta espécie. No Estado do Tocantins, P. ochrogaster foi registrado no Parque Estadual do Cantão (Tocantins 2004a) e no entorno da Ilha do Bananal (Olmos 2003). Entretanto, parece ter uma distribuição muito mais ampla, havendo o provável registro de sua ocorrência no norte do Estado, em Wanderlândia e Santa Fé do Araguaia (SEPLAN 2005), para o vale do rio Paranã (sudeste do Estado) onde é localmente comum (Pacheco \& Olmos 2006) e para a região central, na área de influência da UHE Luis Eduardo Magalhães (R.T. Pinheiro, observação pessoal). Nossos registros mostram que $P$. ochrogaster é comum no PEC, área que provavelmente comporta as maiores populações desta espécie no oeste do Tocantins, sendo vista regularmente e encontrada em toda sua extensão, inclusive nas áreas de uso mais intensivo, formando pequenos bandos ou individualmente (Dornas \& Pinheiro 2007). No entorno (APA), os poucos remanescentes florestais, isolados por áreas de pastagem, tornaram a espécie escassa o que poderia estar restringindo sua ocorrência apenas aos remanescentes florestais de maior tamanho.

Harpyhaliaetus coronatus (Vieillot, 1817) - classificada como vulnerável (MMA 2003), esta espécie foi elevada à categoria de ameaçada pela IUCN em virtude do reduzido número de populações e a distribuição fragmentada das mesmas (IUCN 2006). Na região do Cantão é considerada extremamente rara, sendo avistada em maio de 2005, pousada sob uma árvore seca em área de pastagem na Fazenda Rincão, município de Caseara (09³0' 53" S e 49 51 ' $18^{\circ}$ ” W). No PEC, um indivíduo foi visualizado, em meados de agosto de 2006, em sobrevôo planado, sobre o rio Javaés, próximo ao Centro de Pesquisas Canguçu - UFT (09 $58^{\circ}$ '57' S e 50 00' 54" W). A caça a esta espécie não foi constatada, entretanto, a destruição do hábitat seria o fator de maior risco para a espécie na região.

Harpia harpyja (Linnaeus, 1758) - considerada como quase ameaçada (IUCN 2006), possuía ampla distribuição em todo o Brasil, mas atualmente encontra-se extinto ou quase-extinto em diversas regiões do sul, sudeste e centro-oeste. Sua presença foi registrada através de registros fotográficos em julho de 2005 no Parque Estadual do Cantão, próximo ao furo da Barreirinha (09²4' $13^{\prime \prime}$ S e $\left.50^{\circ} 00^{\prime} 03^{\prime \prime} \mathrm{W}\right)$. Espécie localmente rara, não existindo registros anteriores para o Parque (Tocantins 2004a), APA Bananal/Cantão (MRS 1999) e PARNA Araguaia (MMA/Eletronorte/Proaves 2001). Entretanto, existem registros de um ninho localizado no extremo sul do PEC, construído em árvore de jatobá (Hymenaea stilbocarpa), sob a qual foram encontrados restos de mamíferos de médio porte (paca, cutia e macaco Alouatta sp.) (Roberto Marapiranga, comunicação pessoal).

Alipiopsitta xanthops (Spix, 1824) - papagaio endêmico do Cerrado (Silva 1995, 1997) é dado como globalmente quase ameaçado, principalmente devido à grande modificação antrópica do Cerrado (IUCN 2006). Na área de estudo um único registro foi efetuado de um bando com nove indivíduos, às margens da rodovia TO - 080, na APA, entre os municípios de Marianópolis e Caseara. Ainda que seja escasso na região estudada, a presença de A. xanthops foi assinalada em outras localidades do Estado. Braz e colaboradores (2003) detectaram a espécie na região do Jalapão (à leste) e Olmos et al. (2004) na região de Wanderlândia-Babaçulândia (à nordeste). No sul/sudeste do Estado, Pacheco \& Olmos (2006) descrevem uma numerosa população para a região de Aurora do Tocantins, T. Dornas e R.T. Pinheiro registraram sua presença no município de Rio da Conceição enquanto E.Reis e R.T. Pinheiro no Parque Estadual do Lajeado e R.T. Pinheiro na área urbana de Palmas, região central do Estado, indicando uma ampla distribuição desta espécie no Tocantins.

Celeus torquatus (Boddaert, 1783) - pica-pau amazônico, apresenta subespécie disjunta na Mata Atlântica (Celeus torquatus tinnunculus) onde está sob ameaça de extinção (Sick 1997, MMA 2003). Pacheco \& Olmos (2006) relatam a presença da espécie para o estado do Tocantins em matas ciliares de Aurora do Tocantins (sudeste do Estado), sendo possivelmente o registro mais ocidental da população amazônica. O presente estudo traz o registro de um único individuo, às margens do rio do Côco na porção norte do PEC, próximo ao lago Pesqueirão ( $9^{\circ} 20^{\prime} 26^{\prime}$ ' S e $49^{\circ} 58^{\prime} 29^{\prime \prime} \mathrm{W}$ ). Sua visualização se deu em mata sazonalmente alagada, no extrato de sub-dossel, o qual é indicado como mais utilizado pela espécie (Walther 2002). Os registros efetuados por Tocantins (2004a) verificaram ocorrência de $C$. torquatus na mesma vegetação sazonalmente alagada do Parque Estadual do Cantão.

Synallaxis simoni Hellmayr, 1907 - furnarídeo considerado endêmico da bacia do Araguaia (Silva 1995, 1996, 1997, Sick 1997), com distribuição restrita a sua porção média, é substituído por seu congênere amazônico, S. gujanensis mais ao norte (Olmos et al. 2004). Até recentemente era considerado subespécie de $S$. albilora, proposição desconsiderada devido ao padrão de distribuição da espécie, restrita à planície do médio Araguaia, igualmente a Cercromarca ferdinandi e Paroaria baeri (Silva 1996). Buzzetti (2001) em estudos anteriores no PEC, estudou biometria, bioacústica e alguns aspectos ligados reprodução de $S$. simoni podendo verificar expressivas diferenças acústicas e morfológicas em relação a S. albilora. Assim como ocorrido em Tocantins (2004a), presenciamos e gravamos um indivíduo em ilha fluvial no rio Araguaia $\left(9^{\circ} 15^{\prime} 53^{\prime \prime} \mathrm{S}\right.$ e $\left.50^{\circ} 00^{\prime} 39^{\prime \prime} \mathrm{W}\right)$ e às margens do rio do Côco (9॰21' 23" S e 49 58' 39" W). S. simoni é considerada ameaçado de extinção (MMA 2003, Machado et al. 2005). Segundo o Plano de Manejo do PEC, as atividades turísticas desenvolvidas, nos meses de seca, nas praias das ilhas fluviais do $\mathrm{PEC}$, representam o principal fator de ameaça à presença da espécie (Tocantins 2004b).

Cercomacra ferdinandi Snethlage, 1928 - tem sua distribuição restrita ao Estado do Tocantins e divisa com o Maranhão, ocorrendo na margem leste do rio Araguaia a partir da Ilha do Bananal e a oeste do rio Tocantins no norte do Estado, em tributários de ambos os rios (Olmos et al. 2006). Listada como vulnerável (MMA 2003, IUCN 2006), é relativamente comum em toda a extensão do P. E. Cantão. A supressão da vegetação ribeirinha em função da construção de hidrelétricas é tida como a maior ameaça à espécie (Olmos et al. 2006), entretanto, na área de estudo as ameaças se devem à destruição do habitat promovido pelo incremento das monoculturas e dos 
assentamentos rurais ao longo das margens do rio do Côco, Caiapó e seus afluentes.

Charitospiza eucosma Oberholser, 1905 - considerado endêmico e com ampla ocorrência no Cerrado (Silva 1995, 1996, 1997), é classificado como globalmente quase-ameaçado de extinção, principalmente pela perda de habitats do Cerrado decorrentes dos grandes empreendimentos de pecuária e monocultura (IUCN 2006). Cavalcanti \& Alves (1997) discutem sobre sua especialidade e pioneirismo em colonizar e forragear em ambientes queimados. A ocorrência desta espécie na área de estudo se deu apenas na região da APA, em áreas de cerrado típico, em estradas vicinais próximo ao assentamento Manchete $\left(09^{\circ} 46^{\prime} 75^{\prime}\right.$ ' S e $49^{\circ} 55^{\prime} 03$ " W), não sendo visualizada no PEC. Para o Tocantins, C. eucosma parece ter uma ampla distribuição, possuindo registros por diversas localidades do estado (Braz et al. 2003, Bagno \& Abreu, 2001, Pinheiro 2004, Tocantins 2004a, Pacheco \& Olmos 2006 e Dornas \& Pinheiro 2007 obs. pessoal na Serra das Mangabeiras no município de Rio da Conceição).

\section{Registros novos e importantes}

Informações referentes à Hemitriccus minimus, Heterocercus linteatus, Xenopipo atronitens e Paroaria baeri, encontra-se em Pinheiro e Dornas (submetido) e Sturnella militaris em Dornas et al. (2007).

Egretta caerulea (Linnaeus, 1758) - ardeídeo de coloração violácea (adulto); é a garça mais adaptada aos lamaçais de vazante (Sick 1997), sendo comum em áreas marítimas e manguezais (Sigrist 2006). Rapolle e colaboradores (1993) consideram a possibilidade de migrações oriundas da região neártica para a espécie, no entanto, a presença de populações reprodutivas e residentes na região neotropical é bem marcante. Em janeiro de 2006, foi constatado o primeiro registro para o Parque Estadual do Cantão. Um indivíduo adulto e solitário foi visualizado na praia da Ilha $\left(09^{\circ} 14^{\prime} 02^{\prime}\right.$ ' S e $\left.49^{\circ} 58^{\prime} 37^{\prime \prime} \mathrm{W}\right)$, região de confluência do rio do Côco e rio Araguaia, durante o período de cheia.

Falco peregrinus Tunstall, 1771 - espécie cosmopolita, apresenta populações migratórias neárticas (Rapolle et al. 1993, Sick 1997, Stotz et al. 1996). Sua presença foi constada em abril de 2006, na porção norte do Parque Estadual do Cantão, no topo de árvores de grande porte, às margens do rio Araguaia. Devido ao tamanho avantajado do espécime observado pareceu tratar-se de uma fêmea. Mestre (2006) relata capturas no Brasil de indivíduos com mais de $14.000 \mathrm{~km}$ de distância entre o local de captura e anilhamento, a maioria para estados do sul-sudeste do Brasil. Devido ao período do ano cujo registro foi verificado, este indivíduo provavelmente estava retornando à região neártica para o início do próximo período reprodutivo, utilizando a calha do rio Araguaia como rota migratória.

Bucco tamatia Gmelin, 1788 - espécie inconspícua encontrada nas matas de igapó, várzea e matas alagadas (Sick 1997). Entretanto, sua presença também é dada para florestas de terra firme e campinaranas (Borges et al. 2001). No atual estudo foram visualizados indivíduos da espécie em toda extensão do PEC, sempre associados a áreas de matas alagadas. Ainda que diversos autores (Schauensee \& Phelps 1978, Hilty \& Brown 1986, Sick 1997) limitam a distribuição desta espécie a Amazônia centro-ocidental, este e recentes registros têm confirmado a presença de $B$. tamatia para a região oriental da Amazônia (Tocantins 2004a, Pacheco \& Olmos 2005, Silveira 2006, Hass et al. 2007, Pacheco et al. 2007). Todavia, Pinheiro (2004), apresenta o registro de $B$. tamatia para a região central do estado do Tocantins. Portanto, a ocorrência desta espécie se expande além dos domínios amazônicos, adentrando-se ao bioma Cerrado. A presença das florestas ombrófilas, desde a calha do rio Araguaia, a oeste, até o centro norte do Tocantins, justificam a magnitude da ocorrência de B. tamatia, ainda que tais ambientes encontram-se fortemente fragmentados. Silva (1995) afirma forte dependência deste bucconídeo à habitats florestais, de modo que, os registros de indivíduos da espécie nas florestas ombrófilas da região de Wanderlândia, centro-norte do Tocantins, reafirmam e corroboram a amplitude geográfica de B. tamatia. Entretanto, conforme discutido por Olmos et al. (2004), a destruição deste habitat é rapidamente sentida pela espécie, com o conseqüente declínio e extinção de suas populações.

Myrmotherula multostriata Sclater, 1858 - espécie anteriormente considerada subespécie de $M$. surinamensis, foi recentemente separada como espécie independente (Isler et al. 1999). Possui ampla distribuição ocorrendo do leste Amazônico, desde o litoral paraense até a Amazônia ocidental no Peru, alcançando o sul e sudeste do Pará (Isler et al. 1999, Pacheco et al. 2007). Para o Tocantins existem registros recentes para a região de Wanderlândia (Olmos et al. 2004) e o atual registro para o Parque Estadual do Cantão. Estas duas novas localidades implicam no aumento de sua área de ocorrência, sendo estes os registros mais orientais para a espécie, em relação ao seu centro de distribuição. Importante ressaltar o registro de nidificação da espécie e de uma população bem numerosa no Parque Estadual do Cantão. Esta espécie, fortemente ligada às matas de várzea e igapó (Sigrist 2006), encontra habitats ideais, justificando a sua presença em diferentes áreas alagadas do PEC.

Herpsilochmus longirostris Pelzeln, 1868 - espécie dependente de áreas florestais (Silva 1995, 1997) é considerada endêmica e largamente distribuída para o Cerrado (Silva 1995, Stotz et al. 1996). Willis (1992) discute sua origem a partir da especiação conjunta à H. pectoralis, congênere associadas a áreas da Caatinga. Esta espécie registrada próximo à ilha do Bananal, em áreas florestais do sul do PEC (09 $58^{\circ} 57^{\prime}$ ' S e 50 $00^{\circ}$ '54" W), assim como pelos estudos anteriores (Tocantins 2004a), parece ter no PEC sua ocorrência mais a noroeste para o Cerrado quando comparada a sua área de distribuição geográfica atual (Ridgely \& Tudor 1994, Sick 1997).

Attila cinnamomeus (Gmelin, 1789) - tiranídeo amazônico, prefere as matas de igapó e matas de várzeas, beira de lagos e florestas ribeirinhas (Sick 1997, Borges \& Carvalhães 2000, Sigrist 2006). Esta espécie foi observada por toda a extensão do PEC, sempre associada a regiões alagadas, assim como pelos estudos conduzidos por Tocantins (2004a). Diversos autores (Schauensee \& Phelps 1978, Hilty \& Brown 1986, Ridgely \& Tudor 1994, Sick 1997, Sigrist 1996) relatam a ocorrência desta espécie para quase toda região amazônica, exceto para o leste-sudeste amazônico. Desta maneira, os registros apresentados e a documentação fotográfica para o PEC, somados aos registros dessa espécie para a região de matas ombrófilas no município de Wanderlândia, norte do Tocantins (Olmos et al. 2004) são os mais orientais dentro dos domínios da Amazônia, implicando no aumento da ocorrência da distribuição da espécie até a região de transição com o bioma Cerrado.

Progne subis (Linnaeus, 1758) - oriundas da América do Norte (Sick 1997), migram aos milhares para a América do Sul entre setembro e março, ocorrendo desde a Colômbia até Argentina (Ridgely \& Tudor 1989). No Brasil há registros da espécie para região Amazônica, desde a divisa entre Brasil e Colômbia até o leste do Pará (Pinto 1944 apud Antas et al. 1986, Sick 1997, Borges et al. 2001, Pacheco \& Olmos 2005, Nunes et al. 2006, Silveira 2006). Destacam-se os registros de abundantes bandos para a região de Manaus (Stotz et al. 1992). Para a Caatinga sua ocorrência é prevista no baixo rio São Francisco, a jusante de Juazeiro-BA (Pacheco 2004), e na caatinga maranhense, quase na divisa com Piauí (Nunes et al. 2006). Para o centro-oeste, Antas e colaboradores (1986) descrevem o primeiro registro da espécie para o estado do Mato Grosso, enquanto Tubelis \& Tomas (2000) e Antas (2006) apresentam registros da espécie para o Pantanal do Mato Grosso do Sul e Mato Grosso. Para a região sul/sudeste do país, é relatada a presença da espécie nos estados do Espírito Santo, Rio de Janeiro (Sick 1997) e para o estado de São Paulo, (Sick 1997, 
Antas 2004) onde são constatados concentrações de aproximadamente 70.000 indivíduos (Antas 2006). Recentemente Olmos et al. (2004) detectaram $P$. subis entre Darcinópolis e Aguiarnópolis, na região do médio rio Tocantins, ainda no estado do Tocantins, descobrindo uma nova localidade de ocorrência da espécie. Todavia, em novembro de 2005 registramos enormes bandos de Progne subis pousados em bancos de areias do rio do Côco (09 $9^{\circ} 19^{\prime} 27^{\prime}$ ' S e 49 $\left.9^{\circ} 58^{\prime} 07^{\prime \prime} \mathrm{W}\right)$ dentro do Parque Estadual do Cantão. Este registro coincide em tempo e abundância aos registros efetuados também para Progne subis por Pacheco \& Olmos (2008), os quais são considerados os primeiros para a região. Contudo, no dia 13 dezembro de 2006 um gigantesco bando, com quase uma dezena de milhares de indivíduos (entre 5.000 e 8.000 indivíduos) foi registrado sobre a torre de telefonia da cidade de Caseara $\left(09^{\circ} 16^{\prime} 48^{\prime}\right.$ ' S e $\left.49^{\circ} 57^{\prime} 21^{\prime \prime} \mathrm{W}\right)$. A presença deste bando, nesta antena de telefonia, perdurou por todo o mês de dezembro, sendo ainda marcante a presença de quase mil indivíduos remanescentes, ainda na primeira quinzena de janeiro de 2007. A regularidade dos registros em dois anos consecutivos, afirmam à condição do Parque Estadual do Cantão e da cidade de Caseara como pontos de parada na rota migratória deste migrante neártico em direção as porções mais meridionais do continente.

Compsothraupis loricata (Lichtenstein, 1819) - traupídeo de grande porte é considerado endêmico de ambientes semi-áridos, tendo a Caatinga como centro de ocorrência (Sick 1997). Olmos et al. (1998) relatam a espécie para o norte de Goiás enquanto Pacheco \& Olmos (2006) presenciaram a espécie no sudeste do Tocantins sempre em manchas de mata seca o que favorece a ocorrência desta espécie dentro do bioma Cerrado. Entretanto, Hellmayr (1929) apud Pacheco \& Olmos (2006) relata coletas na região da bacia Araguaia-Tocantins, destacando a presença de $C$. loricata em afloramentos calcários com vegetação de matas secas no alto rio Araguaia. Para o PEC presenciamos $C$. loricata em uma única oportunidade, vocalizando intensamente, por aproximadamente 20 minutos, em ilha fluvial do rio Araguaia ( $9^{\circ} 15^{\prime} 53^{\prime \prime}$ S e $\left.50^{\circ} 00^{\prime} 39^{\prime \prime} \mathrm{W}\right)$, cuja vegetação assemelha-se à fitofisionomias de regiões semi-áridas.

Sporophila castaneiventris Cabanis, 1849 - Emberezídeo tipicamente Amazônico, ocorre em capinzais, principalmente das áreas pantanosas e margens de rios (Schauensee \& Phelps 1978, Hilty \% Brown 1986, Ridgely \& Tudor 1989, Sick 1997). Sua ocorrência se destaca até a região central da Amazônia (Borges et al. 2001), entretanto, novos registros da espécie têm mostrado sua ocorrência no leste amazônico (Tocantins 2004a, Olmos et al. 2004, Pacheco \& Olmos 2005, Silveira 2006). No presente estudo, S. casteneiventris foi observado com freqüência aos casais em ilhas fluviais do rio Araguaia, muitas vezes associados à bandos de $S$. caerulencens e $S$. lineola. Portanto, esta sequiência de recentes registros para $S$. casteneiventris no leste amazônico contribuem de forma considerável para a ampliação da ocorrência desta espécie.

Saltator atricollis Vieillot, 1817 - espécie endêmica do Cerrado (Silva 1995, 1997) vive em grupos pequenos nos campos cerrados e em áreas abertas correspondentes (Sick 1997). Com ampla distribuição por todo bioma Cerrado (Silva 1995, 1997, Fontes et al. 2000, Tubelis \& Cavalcanti 2001, Vasconcelos et al. 2002, Pinheiro 2004) esta espécie foi visualizada em áreas de Cerrado, como também em áreas antropizadas na APA Bananal/Cantão, não sendo registrada para o PEC, provavelmente em função da reduzida representatividade deste habitat.

\section{Discussão}

\section{Diversidade de aves}

A região do Cantão, inserida entre a Amazônia e o Cerrado, comporta uma grande diversidade de aves. O número de espécies da região chega a 418, somando-se as atuais 382 às 36 incluídas na Avaliação Ecológica Rápida que subsidiou o Plano de Manejo do PEC (Tocantins 2004a), e que não foram observadas neste trabalho (Tabela 1). Este número pode ser ainda maior, uma vez que, áreas de grande importância, como o delta e interior do rio Caiapó, os grandes remanescentes de Floresta Ombrófila e Semidecidual do município de Caseara e Araguacema, assim como os capões de mata natural (localmente conhecidos como Ipucas) localizados nos campos de murundus do município de Pium, e as matas ciliares do rio do Côco e seus afluentes localizados fora dos limites do Parque, não foram satisfatoriamente explorados. A região sul do Parque Estadual do Cantão também merece especial atenção, uma vez que ali se concentram extensas áreas de mata contínua e em satisfatório estado de conservação.

O PEC exerce um importante papel na preservação de diversas espécies ameaçadas. As populações de espécies vulneráveis à extinção como Cercomacra ferdinandi encontram no PEC uma área fundamental para sua preservação, sendo potencialmente o local com os principais efetivos populacionais conhecidos na sua região de distribuição. Destaca-se ainda a presença de Synallaxis simoni, furnariídeo de distribuição restrita ao vale do rio Araguaia (Silva, 1997), cujos registros recentes foram feitos todos no PEC (Buzzetti 2001, Tocantins 2004a, este estudo), ou ainda uma nova espécie de furnarídeo do gênero Certhiaxis que está sendo descrita (Dante Buzzetti comunicação pessoal).

Outra importante espécie, Penelope ochogaster, ameaçada de extinção (MMA 2003), apresenta iminente risco em função da atividade turística desordenada vigente no PEC, pela supressão da cobertura vegetal e pela pressão de caça na região. Este cracídeo utiliza as margens arenosas dos rios e canais em busca de alimento, ambientes que nos meses centrais do ano estão repletos de turistas e pescadores. Dornas \& Pinheiro (2007), estudando as populações de P. ochrogaster do PEC, encontraram, durante os meses centrais do ano (junho a agosto), uma maior abundância da espécie nas praias ribeirinhas do rio Javaés, extremo sul do Parque, em relação às porções mais setentrionais dos rios Araguaia e do Côco no PEC. Isto sugere que o uso intensivo das áreas ribeirinhas no setor norte do Parque pela população local e turistas, poderia ser uns dos fatores inibidores da presença desta espécie, ressaltando ainda que, o somatório destes fatores poderia favorecer o seu desaparecimento localmente.

O estudo revelou que várias outras espécies amazônicas penetram no bioma do Cerrado seguindo as florestas de galeria do Cerrado, tal como foi sugerido por Silva (1996). Tal descoberta resulta em importantes implicações ecológicas e biogeográficas, pois reforça o papel da região do Cantão como uma zona de tensão ecológica entre os a Amazônia e o Cerrado.

Silva \& Santos (2005) adicionaram 19 espécies à lista de aves do bioma do Cerrado após os estudos de Silva (1995), aumentado a lista da região para 856 espécies. O presente estudo adicionou mais oito novas espécies: Hydropsalis climacocerca, Lophornis gouldii, Inezia subflava, Knipolegus poecilocercus, Atilla cinnamomeus, Hylophilus semicinereus, Ammodramus aurifrons e Sturnella militaris (detalhes desta última em Dornas et al. 2007), aumentando, portanto, este total para 864 espécies de aves presentes nos limites do bioma Cerrado.

\section{Prioridades de conservação e recomendações}

A elevada diversidade de aves da região do Cantão depende da integridade dos diversos hábitats ali presentes e que são únicos em todo o Estado. Entretanto, a integridade dos ecossistemas no Parque Estadual do Cantão e da Área de Proteção Ambiental Ilha do Bananal/Cantão parece ser um reflexo direto do uso legal conferido a estas duas categorias, sendo, portanto, mais íntegras no PEC (UC de Proteção Integral) e mais alteradas na APA (UC de uso sustentado). 
Apesar das restrições impostas pela legislação, a presença de inúmeras áreas transformadas em roças de toco, onde se cultiva mandioca e grãos (arroz, feijão, milho), e a permanência de algumas famílias residentes no interior do Parque, alterou consideravelmente a paisagem do extremo norte do PEC. Estas transformações têm favorecido a penetração de diversas espécies da flora e fauna típicas de ambientes abertos e semi-abertos, fazendo com que a proporção de espécies de aves com esta preferência de habitat seja semelhante àquelas da APA, onde predominam as fitofisionomias mais abertas do cerrado. A integral regularização fundiária deve ser priorizada, erradicando em definitivo, o uso das terras interiores para este fim, de maneira que a vegetação típica local se restabeleça, minimizando outros impactos à fauna e flora local.

Nos municípios do entorno do Parque que compõe a APA, a pecuária tem um papel histórico na fragmentação da paisagem do Cerrado predominante na região. A situação torna-se mais alarmante com a intenção do governo estadual em reduzir a área da APA Ilha do Bananal/Cantão para a ampliação das áreas de cultivo de arroz irrigado, monocultura de grãos, cana-de-açúcar e oleaginosas. Estas duas últimas já vêm, de forma tímida, alterando a paisagem local. Como resultado final presume-se uma grande interferência no fluxo da biota local, uma vez que, no período das cheias, a maioria das espécies aquáticas, dentre elas o pato-corredor (Neochen jubata) busca refúgio nas várzeas transformadas em monoculturas de arroz irrigado, ricas em alimento, localizadas nos municípios do Pium e Lagoa da Confusão.

É significativo o registro de diversas espécies endêmicas e/ou ameaçadas/quase-ameaçadas na APA (Harpyhaliaetus coronatus, Penelope ochrogaster, Alipiopsitta xanthops, Melanopareia torquata, Charitospiza eucosma, Saltator atricollis), entretanto, o incremento das alterações nas paisagens naturais da APA, podem tornar as populações destas e de outras espécies - aqui não somente as aves - ainda mais vulneráveis em termos de conservação.

Por outro lado, são os ambientes florestais os mais ameaçados dentro dos limites da APA. Os inúmeros assentamentos rurais, estabelecidos praticamente sem nenhuma análise de impacto ambiental, são implantados nos escassos remanescentes florestais da região. Áreas de Floresta Ombrófila e Florestas Sazonalmente Alagadas, típicas das formações Amazônicas, são fragmentadas e áreas extensas suprimidas rapidamente conforme constatado. Desta maneira, espécies tipicamente amazônicas registradas somente nestas formações (Terenotriccus erythrurus, Lipaugus vociferans, Pipra rubrocapilla, entre outros), correm sério risco de extinguir-se localmente. A preservação das espécies fora dos limites do Parque é ainda mais preocupante, uma vez que, muitos remanescentes florestais estão localizados em áreas de assentamento do INCRA, impossibilitando a sua transformação em unidades de conservação.

Em face às graves ameaças aos ecossistemas e a vulnerabilidade de algumas espécies da avifauna local, recomenda-se a implantação de novas Unidades de Conservação de Proteção Integral que contemplem as áreas de Florestas Ombrófilas às margens do rio Caiapó, as Ipucas (capões de mata envoltos por vasta matriz de Cerrado) e varjões localizados nos município de Pium e Lagoa da Confusão, assim como as áreas de Floresta Sazonalmente Alagada às margens do rio Araguaia, entre os municípios de Araguacema e Caseara, as quais ainda apresentam remanescentes em bom estado de conservação.

A região do Cantão possui características únicas e que devem ser preservadas. É uma área em constante tensão ecológica, dotada de grande diversidade biológica, fruto da transição entre o Cerrado e a Floresta Amazônica. Ao estar localizada no vale do Araguaia, destaca-se ainda por ser rica em endemismos. Entretanto, somente através de ações de manejo e conservação, e de estudos detalhados é que obteremos informações sobre a sustentabilidade de algumas populações de aves, principalmente aquelas ameaçadas, a médio e longo prazo na região.

\section{Agradecimentos}

Agradecemos a Joaquim Carneiro por acompanhar e guiar-nos em campo, aos colegas Deborah Rodello, Divino Nunes, Elivânia Reis, Geisa Goulart, Giselly Cintra, Magno Carneiro, Marcelo Barbosa, Maria Amélia F. Maciel, que nos acompanharam em campo, ao Instituto Natureza do Estado do Tocantins - Naturatins e a Gerência do Parque Estadual do Cantão, pelo importante apoio logístico e ainda à Conservação Internacional do Brasil, pelo financiamento do projeto Conservação de Aves Migratórias no Brasil e à Fundação ULBRA - FULBRA. O mapa com a localização da área de estudos foi elaborado por Maria Amélia F.Maciel.

\section{Referências Bibliográficas}

ANTAS, P.T.Z., YAMASHITA, C. \& VALLE, M.P. 1986. First Record of Purple Martin (Progne subis) in Mato Grosso State, Brazil. J. f. ornith. 57(2):171-172.

ANTAS, P.T.Z. 2004. Pantanal - Guia de Aves: espécies de aves da Reserva do Patrimônio Natural do SESC Pantanal. SESC, Departamento Nacional, Rio de Janeiro. 246p.

BAGNO, M.A. \& ABREU, T.L.S. 2001. Avifauna da região da Serra do Lajeado, Tocantins. Humanitas. 3:51-70.

Birdlife International. 2006. Species factsheet: Neochen jubata. Available from: <http://www.birdlife.org > (último acesso em 02/01/07).

BORGES, S.H. \& CARVALHÃES, A. 2000. Bird species of black water inundation forests in the Jaú National Park (Amazonas State, Brazil): their contribution to regional species richness. Biodiv. cons. 9:201-214.

BORGES, S.H., COHN-HAFT, M., CARVALHAES, A.M.P., HENRIQUES, L.M., PACHECO, J.F. \& WHITTAKER, A. 2001. Birds of the Jaú National Park, Brazilian Amazon: species check-list, biogeography and conservation. Ornit. neot. 12(2):109-140.

BRAZ, V.S., ABREU, T.L.S., LOPES, L.E., LEITE, L.O., FRANÇA, F.G.R., VASCONCELLOS, M.M. \& BALBINO, S.F. 2003. Brazilian Merganser Mergus octosetaceus discovered in Jalapão State Park, Tocantins, Brazil. Cotinga. 20:68-71.

BUZZETTI, D. 2001. Novas informações sobre a Ecologia de Synallaxis simoni, taxon endêmico da bacia do rio Araguaia. In IX Congresso Brasileiro de Ornitologia (Editor Fernando C. Straube). Curitiba, p. 156-157.

CAVALCANTI, R.B. \& ALVES, M.A.S. 1997. Effects of fire on savanna birds in Central Brazil. Ornit. neot. 8(1):85-87.

Comitê Brasileiro de Registros Ornitológicos. 2007. Listas das aves do Brasil. Versão 16/8/2007. Available from: <http://www.cbro.org.br>. (último acesso em 10/04/2008).

DORNAS, T. \& PINHEIRO, R.T. 2007. Dinâmica populacional de Penelope ochrogaster em ambientes fluviais do Parque Estadual do Cantão, Tocantins. In XV Congresso Brasileiro de Ornitologia. (Editora Carla Suertegaray Fontana). Editora EDIPUCRS, Porto Alegre, p. 201-202.

DORNAS, T., PINHEIRO, R.T., PACHECO, J.F. \& OLMOS, F. 2007. Ocorrência de Sturnella militaris (Linnaeus, 1758), polícia-inglesado-norte no Tocantins e sudoeste do Maranhão. Rev. bras. ornit. 15(3):448-450.

FONTES, J., CINTRA, R. \& SANAIOTTI, T. 2000. Efeito da variação na composição da comunidade de árvores na riqueza e abundância de aves em um Cerrado Mato-Grossense. In III Simpósio sobre Recursos Naturais e Sócio-Econômicos do Pantanal. EMBRAPA, Corumbá, p. 1-13.

HASS, A., PAULA, W.S. \& BARRETO, L. 2007. Caracterização da avifauna da região de Balsas In: Cerrado Norte do Brasil (L. Barreto, org.). Useb, Pelotas, 379p.

HIDASI, J. 1968. Notas Zoológicas da Ilha do Bananal, Goiânia. Dep. Estadual de Cultura Estado de Goiás, Goiânia, p. 28. 
HIDASI, J. 1983. Lista preliminar das aves do Estado de Goiás. Fundação Museu Ornitológico de Goiânia, Goiânia.

HIDASI, J. 1998. Lista preliminar das aves do Tocantins. Fundação da Universidade do Tocantins - Unitins, Palmas.

HILTY, S.L. \& BROWN, W.L. 1986. A guide to the birds of Colombia. Princeton University Press, Princeton.

ISLER, M.L., ISLER, P.R. \& WHITNEY B.M. 1999. Species limits in antbirds (Passeriformes: Thamnophilidae): the Myrmotherula surinamensis complex. The Auk. 116(11):83-96.

The World Conservation Union - IUCN. 2006. IUCN Red List of Threatened Species. Available from: <http://www.iucnredlist.org/search/details. php/8966/summ>. (último acesso em 10/01/07).

KLINK, C.A. \& MACHADO, R.B. 2005. Conservation of the Brazilian Cerrado. Cons. biol. 19(3):707-713.

MACHADO, A.B.M., MARTINS, C.S. \& DRUMMOND, G.M. 2005. Lista da fauna brasileira ameaçada de extinção. Fundação Biodiversitas, Belo Horizonte, 160p.

MESTRE, L.A.M. 2006. Migrações de Falco peregrinus anilhados na América do Norte e recapturados no Brasil entre 1967 e 2001. In XIV Congresso Brasileiro de Ornitologia (Editor Rômulo Ribon). Ouro Preto, Seção Faunística, p. 31.

Ministério do Meio Ambiente - MMA. 2003. Lista das espécies de fauna ameaçada de extinção. Available from: <http://www.ibama.gov.br/fauna/ downloads/lista\%20spp.pdf>. (último acesso em 02/01/07).

MMA/SBF. 2002. Biodiversidade Brasileira: avaliação e identificação de áreas e ações prioritárias para conservação, utilização sustentável e repartição de benefícios da biodiversidade brasileira. Brasília, 404p.

MMA/ELETRONORTE/PROAVES. 2001. Plano de Manejo do Parque Nacional do Araguaia. Brasília, 133p.

MRS. 1999. Plano de Gestão da Área de Proteção Ambiental Ilha do Bananal/ Cantão. Relatório não publicado.

NAKA, L.N., RODRIGUES, M., ROOS, A.L. \& AZEVEDO, M.A.G. 2002. Bird Conservation on Santa Catarina Island, Southern Brazil. Bird cons. intern. 12:123-150.

NUNES, M.F.C., LACERDA, R., ROOS, A. \& COSTA, J. 2006. Aves Migratórias na Amazônia e a Gripe Aviária. Centro Nacional de Pesquisa para Conservação das Aves silvestres. Available from: <http://www. fmt.am.gov.br/imprensa/aves \%20migratorias \%20amazonia\%20e\%20 gripe\%20aviaria.pdf > . (último acesso em 12/02/2007).

OLMOS, F., MARTUSCELLI, P. \& SILVA, R.S. 1998. Ecology and habitat of Pfrimer's Conure Pyrrhura pfrimeri, with a reappraisal of Brazilian Pyrrhura leucotis. Ornit. neot. 8(2):121-132.

OLMOS, F. 2003. The Chestnut-bellied Guan Penelope ochrogaster in the Araguaia valley, Tocantins. Cotinga. 20:64-65.

OLMOS, F., ARBOCZ, G., PACHECO, J.F. \& DIAS, R.R. 2004. Estudo da flora e Fauna do Norte do Estado do Tocantins (R. R. Dias, org.). Secretaria do Planejamento e Meio Ambiente, Diretoria de Zoneamento EcológicoEconômico, Palmas. Projeto de Gestão Ambiental Integrada da Região do Bico do Papagaio. Zoneamento Ecológico-Econômico.

OLMOS, F., SILVA, R.S., PACHECO, J.F. 2006. The range of the Bananal Antbird Cercomacra ferdinandi. Cotinga. 25:21-23.

OLMOS, F., PACHECO, J.F. 2008. Large Puple Martin Progne subis Roosts in the Araguaia Valley, Central Brazil. Cotinga. 29:117.

PACHECO, J.F. 2004. As aves da Caatinga: uma análise histórica do conhecimento. In Biodiversidade da Caatinga: áreas e ações prioritárias para conservação (J.M.C. Silva, M. Tabarelli, M.T. Fonseca \& L.V. Lins, eds.). MMA, Brasília. p. 189-250.

PACHECO, J.F. \& OLMOS, F. 2005. Birds of a Latitudinal Transect in the Tapajós-Xingu Interfluvium, eastern Brazilian Amazônia. Ararajuba. 13(1):29-46.

PACHECO, J.F. \& OLMOS, F. 2006. As aves do Tocantins 1: região sudeste. Rev. bras. ornit. 14:55-71.

PACHECO, J.F., KIRWAN, G.M., ALEIXO, A., WHITNEY, B.M., WHITTAKER, A., MINNS, I., ZIMMER, K.J., FONSECA, P.S.M.,
LIMA, M.F.C. \& OREN, D.C. 2007. An avifaunal inventory of the CVRD Serra dos Carajás project, Pará, Brazil. Cotinga. 27:15-30.

PINHEIRO, R.T. 2004. Captura e recaptura de aves na Região Central do Tocantins, Palmas. Humanitas. 4-6:39-54.

PINHEIRO, R.T. \& DORNAS, T. In press. Novos registros ornitológicos para o Parque Estadual do Cantão: extensão de distribuição e implicações conservacionistas para avifauna do ecótone Amazônia - Cerrado. Rev. bras. ornitol. (submetido)

RIDGELY, R.S. \& TUDOR, G. 1994. The birds of South America. Vol. 2- The Suboscine Passerines. University of Texas Press, Austin.

RAPPOLE, J.H., MORTON E.S., LOVEJOY, T.E. \& RUOS, J.L. 1993. Aves Migratorias Nearticas en los Neotropicos. Smithsonian Institution, CRC.

SANTOS, E.R. \& LOLIS, S.F. 2007. Análise Florística em Comunidades Florestais nos Municípios de Caseara, Marianópolis e Pium, no estado do Tocantins. Carb. soc. 1(02):24-31.

SCHAUENSEE, R. \& PHELPS, W.H. 1978. A guide to the birds of Venezuela. Princeton University Press, Princeton.

Secretaria de Planejamento e Meio Ambiente - SEPLAN. 2005. Atlas do Tocantins: subsídios ao planejamento da gestão territorial. Diretoria de Zoneamento Ecológico-Econômico, Palmas.

SICK, H. 1997. Ornitologia Brasileira. Editora Nova Fronteira, Rio de Janeiro. 912p.

SICK, H. 1983. Migrações de aves na América do Sul continental. IBDF, Brasília. 86p.

SIGRIST, T. 2006. Aves do Brasil, uma visão artística. Fosfertil, Avis Brasilis, São Paulo.

SILVA, J.M.C. 1995. Birds of the Cerrado Region, South America. Steentrupia. 21(2):69-92

SILVA, J.M.C. 1996. Distribuition of Amazonian and Atlantic birds in gallery forest of the Cerrado region, South America. Ornit. neot. 7(1):1-18.

SILVA, J.M.C. 1997. Endemic bird species and conservation in the Cerrado Region, South America. Biodiv. cons. 6:435-450.

SILVA, J.M.C. \& BATES, J.M. 2002. Biogeographic patterns and conservation in the South American Cerrado: A tropical savanna Hotspot. BioScience. 52(3):225-233.

SILVA, J.M.C. \& SANTOS, M.P.D. 2005. A importância relativa dos processos biogeográficos na formação da avifauna do Cerrado e de outros biomas brasileiros. In Cerrado: Ecologia, Biodiversidade e Conservação (A. SCARIOT, J.C. SOUSA FILHO \& J.M. FELFILI, org.). Ministério do Meio Ambiente, Brasília, p. 224-233.

SILVEIRA, L.F. 2006. Diversity of birds and monitoring of cynegetic species in the forest reserves of the Agropalma group, in Tailândia municipality, state of Pará. Technical Report to Agropalma, Pará. Available from: $<$ http://www.ib.usp.br/ lfsilveira/agropalma2.pdf>. (último acesso em $12 / 02 / 2007)$.

SNETHLAGE, E. 1928. Novas espécies e subespécies de aves do Brasil Central. Bol. mus. nac. 4(2):1-7.

STOTZ D.F., BIERREGAARD, R.O., COHN-HAFT, M., PETERMANN, P., SMITH, J., WHITTAKER, A. \& WILSON, S.V. 1992. The status of North American migrants in Central Amazonian Brazil. The Condor. 94:608-621.

STOTZ, D.F., FITZPATRICK, J.W. \& PARKER III, T.A. 1996. Neotropical Birds: ecology and conservation. University of Chicago Press, Chicago, Illinois.

TOCANTINS. 2004a. Avaliação Ecológica Rápida do Parque Estadual do Cantão. Secretaria do Planejamento e Meio Ambiente do Estado do Tocantins, Palmas.

TOCANTINS. 2004b. Plano de Manejo do Parque Estadual do Cantão. Secretaria do Planejamento e Meio Ambiente do Estado do Tocantins, Palmas.

TUBELIS, D.P. \& TOMAS, W.M. 2000. Revisão e atualização da listagem das espécies de aves da planície do Pantanal. In III Simpósio sobre Recursos Naturais e Sócioeconômicos do Pantanal. Embrapa, Corumbá. Available 
from: <http://www.cpap.embrapa.br/agencia/congresso/Bioticos/ TUBELIS-077.pdf>. (último acesso em 25/02/07).

TUBELIS, D.P. \& CAVALCANTI, R.B. 2001. Community similarity and abundance of bird species in open habitats of a central Brazilian Cerrado. Ornit. neot. 12(1):57-73.

VASCONCELOS, M.F., NETO, S.D., BRAND, L.F.S., VENTURIN, N., OLIVEIRA-FILHO, A.T.F. \& COSTA, A.F. 2002. Avifauna de Lavras e municípios adjacentes, sul de Minas Gerais e comentários sobre sua conservação. Unimontes Cient. Montes Claros. 4(2):1-14.
WALTHER, B.A. 2002. Vertical stratification and use of vegetation and light habitats by Neotropical forest birds. J. ornithol. 143:64-81.

WILLIS, E.O. 1992. Zoogeographical origins of eastern Brazilian birds. Ornit. neot. 3(1):1-15.

Recebido em 28/09/07 Versão reformulada recebida em 10/10/08 Publicado em 12/03/09 
